\title{
Mapas de la modernidad: disciplinas espacio-tiempo
}

\author{
Maps of Modernity: \\ Time-Space Disciplines
}

\section{SAURABH DUBE*}

Resumen: Este ensayo se proyectó como una narración personal y desenmaraña cómo llegué a atisbos e indicios sobre el tiempo y el espacio, lo familiar y lo extraño, como parte de un estudio que explora la modernidad, las disciplinas y sus interacciones. Con base en la antropología, la historia y la teoría social y política, considera de manera cuidadosa las contradicciones y las contenciones que atraviesan los estudios subalternos y las perspectivas decoloniales. En conjunto, exploro narraciones usuales sobre un tiempo homogéneo (que, sin embargo, se fundamentan en rupturas inaugurales, espaciales) regidas por modelos contradictorios del espacio social (que, no obstante, implican una jerarquía singular, temporal).

Palabras clave: modernidad; temporalidad; espacialidad; subalternidad; descolonización.

Abstract: This essay is cast as a personal narrative. It unravels how I arrived at inklings and intimations of time and space, familiar and strange, as part of a study that explores modernity, the disciplines, and their interplay. Based in anthropology, history, and socialpolitical theory, the essay carefully considers the contradictions and contentions that run through subaltern studies and decolonial perspectives. Together, I explore routine portrayals of homogeneous time (that are yet founded on inaugural, spatial ruptures) as bound

Recepción: 19 de agosto de 2016./Aceptación: 28 de febrero de 2017.

*El Colegio de México, saurabhdube99@gmail.com 
to antinomian blueprints of social space (which nonetheless entail a singular, temporal hierarchy).

Key words: modernity; temporality; spatiality; subalternity; decoloniality.

El presente artículo forma parte de un proyecto mayor que explora temas de modernidad e historia, basado en consideraciones críticas del tiempo, el espacio y sus engranajes. Utilizando sujetos socioespaciales y temporalidades desarregladas, el proyecto analiza los encantamientos, las oposiciones, las incitaciones y las ambivalencias que constituyen la modernidad. ${ }^{1}$ Aquí se encuentran procedimientos generalizados de la temporalización del espacio y la espacialización del tiempo basados en representaciones de rutina de tiempo homogéneo (que, no obstante, se fundan en rupturas inaugurales y espaciales) y en planos antinomistas del espacio social (que, sin embargo, implican una jerarquía temporal única) que se atan mutuamente. Este ensayo, relatado como narrativa personal, desentraña la manera como llegué a adquirir vislumbres e insinuaciones del tiempo y el espacio, familiares y extraños, arraigados en los estudios subalternos, la antropología histórica, las perspectivas poscoloniales y los entendimientos decoloniales.

\section{Primeras consideraciones}

Al hablar sobre el tiempo y el espacio me preocupan las dimensiones sociales de dichas categorías y procesos. El espacio social y el tiempo social, íntimamente entrelazados el uno con el otro, están lejos de ser contextos meramente pasivos, telones de fondo fácilmente determinados y conductos ya recibidos para la acción humana. Más bien, está a consideración la incesante

${ }^{1}$ En verdad, más que abordar tales antinomias, atracciones y ambigüedades -indicando errores analíticos o carencias históricas que aguardan su corrección (con el tiempo) o (inexorable) superación-, mi intención es, de manera crítica pero cautelosa, desdoblar estos elementos como formadores de mundos modernos. Saurabh Dube, Subjects of Modernity: Time-Space, Disciplines, Margins, Manchester, Manchester University Press, 2017. 
interacción entre entendimientos culturales rutinarios, representaciones ideológicas dominantes y cargadas producciones diarias de espacio y tiempo como constituyentes de las convenciones sociales y las prácticas históricas que las apuntalan y, a la vez, están conformadas por ellas. Dicho de otro modo, tiempo y espacio, elaborados en conjunto, son componentes críticos y resultados activos, atributos formativos y consecuencias clave de sentido y poder, de alteridad y autoridad, y de práctica y proceso que definen los mundos sociales y sus divisiones. ${ }^{2}$

En este ensayo atenderé los intercambios activos entre entendimientos usuales, representaciones hegemónicas y construcciones cotidianas de espacio y tiempo, especialmente en las elaboraciones en estudios subalternos, perspectivas poscoloniales y entendimientos decoloniales. ${ }^{3}$ Por un lado, mi relación

${ }^{2}$ Estos entendimientos del tiempo y el espacio aprovechan y reúnen los énfasis clave de una gama de estudios académicos críticos, escritos que desentrañan la producción del espacio, especialmente bajo el capitalismo; las críticas de los usos disciplinarios del tiempo y las expresiones diarias del espacio y el tiempo en diversas culturas, sociedades e historias. Proporciono muy pocas referencias indicativas en este sentido, pero registro que una discusión sobre los aspectos en que estos análisis difieren entre sí -y las formas en las que pongo sus énfasis a trabajar en mis argumentos- bien sería motivo para otro capítulo, quizá incluso un libro entero. Henri Lefebvre, The Production of Space, Oxford, Blackwell, 1991; Edward Soja, Postmodern Geographies: The Reassertion of Space in Critical Social Theory, Londres, Verso, 1989; Johannes Fabian, Time and the Other: How Anthropology Makes its Object, Nueva York, Columbia University Press, 1983; Dipesh Chakrabarty, Provincializing Europe: Postcolonial Thought and Historical Difference, Princeton, Princeton University Press, 2000; Nancy Munn, "The Cultural Anthropology of Time: A Critical Essay”, Annual Review of Anthropology, vol. 21, 1992, pp. 93-123; Nancy Munn, The Fame of Gawa: A Symbolic Study of Value Transformation in a Massim (Papua New Guinea) Society, Durham, Duke University Press, 1992. Véase también Pierre Bourdieu, Outline of a Theory of Practice, Cambridge, Cambridge University Press, 1977; Michel de Certeau, The Practice of Everyday Life, tr. Steven Rendall, Berkeley, University of California Press, 1984; Reinhart Koselleck, The Practice of Conceptual History: Timing History, Spacing Concepts, tr. Todd Samuel Presner, Stanford, Stanford University Press, 2002, y Nicholas Thomas, Out of Time: History and Evolution in Anthropological Discourse, Cambridge, Cambridge University Press, 1989.

${ }^{3}$ Mientras que las primeras dos tendencias serían familiares para los lectores de esta revista, las perspectivas decoloniales no siempre son tan bien conocidas en el mundo angloparlante. Por este motivo, brindo una introducción básica a los estudios de la colonialidad/decolonialidad durante el presente ensayo. Al mismo tiempo, para un entendimiento de cómo abordo los estudios subalternos y las perspectivas poscoloniales -incluidas las superposiciones y las distinciones entre ellas-, así como la elaboración más amplia de estas tendencias, refiero al lector interesado a Saurabh Dube, "Critical Crossovers: Cultural Identities, Postcolonial Perspectives, and Subaltern Studies", en Margaret Wetherell y Chandra Talpade Mohanty (eds.), The Sage 
crítica con estas perspectivas particulares busca subrayar la centralidad del espacio-tiempo en la creación de los imaginarios sociales modernos. Aquí son importantes en particular los excesos y las ambivalencias aunadas a las contradicciones y los argumentos que acompañan la interpretación cotidiana del tiempo y el espacio en la práctica epistémica en general. Por otro lado, para sostener mis afirmaciones debo entrar, necesaria y cuidadosamente, en los protocolos de los argumentos de los estudios subalternos y los entendimientos decoloniales, desmenuzar sus afirmaciones comunes y tamizarlas también contra sus propias presunciones. Una lectura de tal naturaleza, que a la par va con $y$ contra la veta analítico-epistémica de estos formidables corpus, consciente de sus términos y texturas familiares, pero también atenta a sus giros y vuelcos desconocidos, simplemente no puede evadir lo que Michel de Certeau ha llamado los "detalles". ${ }^{4} \mathrm{Mi}$ esfuerzo es incluir y presentar tales detalles (en ocasiones densos) de manera accesible, guiando al lector interesado hacia textos explicativos sobre estas cuestiones, principalmente en las notas. Así, doy seguimiento a la presencia de lo común, lo hegemónico y lo extraño en la producción analítica y la interpretación diaria del espacio y el tiempo en la práctica epistémica, subrayando igualmente proyecciones persistentes de poder y diferencia, autoridad y alteridad, en estos ámbitos.

Antes debo aclarar tres puntos. En primer lugar, el siguiente recuento no es prescriptivo en forma alguna. Es decir, no estoy sugiriendo que los estudios subalternos, las perspectivas decoloniales o los entendimientos poscoloniales debieron hacer las cosas de otro modo o que la suya es una historia de oportunidades perdidas. El asunto es que tanto la perspectiva subalterna como la decolonial y poscolonial se han manifestado y hay mucho que aprender de los términos en los que sucedieron. En segundo lugar, me interesa cómo estos mismos entendimientos han sido desentrañados a través del tiempo-espacio, incluidos de manera generacional -sus giros, anhelos y vuelcos, sus apari-

Handbook of Identities, Londres, Sage, 2010, pp. 125-143, y Saurabh Dube, Stitches on Time: Colonial Cultures and Postcolonial Tangles, Durham, Duke University Press, 2004, pp. 129-163. Véase también Dube, Subjects of Modernity, op. cit., cap. 1.

${ }^{4}$ Certeau, The Practice of Everyday Life, op. cit., p. ix. 
ciones, por así decirlo-.${ }^{5}$ Esto también significa que deseo analizar las contradicciones y las ambigüedades de los estudios subalternos y las perspectivas decoloniales, pero no como errores analíticos. Más bien, considero tales silencios y ambivalencias como formativos y productivos, que definen las condiciones y las posibilidades de éstas (y otras) perspectivas que deben tenerse en mente al leer el ensayo. Dicho de otro modo, entender no tiene que ver sólo con análisis musculares y descartes fáciles, sino con que a uno le importe conocer. Tal sentido de importancia y conocimiento a la vez está vinculado a aprender y desaprender. Por último, mis historias no buscan la novedad; estudio las transacciones mucho más rutinarias de los mundos intelectuales. En este sentido, cualquier novedad que pueda aparecer más adelante surge de las regularidades y las irregularidades propias de la producción de conocimiento diario. De hecho, mi caminar por lo rutinariamente familiar y desconocido sigue aprendiendo del radical Durkheim, quien sugirió que es en los mundos sociales mundanos donde puede imaginarse lo inimaginable. ${ }^{6}$

${ }^{5} \mathrm{La}$ dimensión generacional es especialmente importante para los estudios subalternos, como notó Ranajit Guha hace casi 20 años. Guha nació a inicios de la década de 1920, una época de dominio imperial: él marcó la primera generación del emprendimiento de los estudios subalternos. Los "hijos de la media noche", casi todos nacidos cerca de la época de la independencia india, que eran parte del colectivo original, fueron la segunda generación de los estudios subalternos. Aunque nunca formé parte del colectivo, que se amplió a mediados de la década de 1990, Dipesh Chakrabarty generosamente me incluyó en la tercera generación de estudios subalternos, junto con sus nuevos miembros, como Ajay Skaria y M. S. S. Pandian; todos nosotros habíamos alcanzado la madurez cuando el binario de colonia y nación había comenzado a marchitarse y desbaratarse. El punto es que el juego de "generación" permea la emergencia estratificada de los estudios subalternos - los argumentos y los énfasis, los términos y las texturas del proyecto- que este ensayo explora. Ranajit Guha, "Introduction", en Ranajit Guha (ed.), A Subaltern Studies Reader: 1986-1995, Minneapolis, University of Minnesota Press, 1997, pp. ix-xv.

${ }^{6}$ Asimismo, todo esto debe aclarar que, contrario a lo que asumió uno de los dictaminadores de este ensayo, mi objetivo en el presente no es analizar el impacto de la perspectiva subalterna, poscolonial y decolonial sobre el academicismo, especialmente en Latinoamérica. No obstante, he discutido tales cuestiones en otros textos. Véase especialmente Saurabh Dube, Modernidad e historia, México, El Colegio de México, 2011. 


\section{Primeros encuentros}

Nacido de padres antropólogos, crecí en Sagar (India central), en Delhi (la nueva y la antigua) y en Shimla (en el norte de India). Mis años formativos estuvieron permeados con un sentido persistente de cómo los terrenos (o los tiempos/espacios) de lo "vernáculo" y lo "cosmopolita” siempre se superponían, pero sólo se encontraban mutuamente de maneras curiosas, particulares y contradictorias. Un poco más adelante, mientras estaba en la búsqueda de mi vocación en la investigación y la enseñanza, realicé estudios en historia, pero me vi atraído por la antropología, especialmente cuando di mis primeros pasos de preaprendiz de académico en el emprendimiento de los estudios subalternos. (Aquí inicio una metáfora artesanal sobre la llegada a la madurez que apuntala la narrativa.)

A partir de la segunda mitad de la década de 1970 se lanzaron innovaciones críticas en la historia del subcontinente. Si bien las reevaluaciones de los pasados del nacionalismo indio con frecuencia fueron centrales en tales emprendimientos, se ofrecían igualmente otras convergencias de importancia. Las lecturas imaginativas de materiales históricos fueron particularmente sustanciales: desde archivos convencionales, incluidos reportes de administradores coloniales, hasta etnografías más tempranas como fuentes de historia, y desde registros vernáculos de la historia previamente vilipendiados hasta diversas expresiones subalternas del pasado. Tales lecturas podrían problematizar la naturaleza misma del archivo histórico e iniciar conversaciones con otras orientaciones, incluidas las de la lingüística estructural y la teoría crítica. ${ }^{7}$ No menos trascendentes fueron los reconocimientos incipientes del carácter innatamente político de la historiografía.

7 Ranajit Guha, “The Prose of Counter-insurgency”, en Ranajit Guha (ed.), Subaltern Studies II: Writings on South Asian History and Society, Delhi, Oxford University Press, 1983, pp. 1-42; Ranajit Guha, Elementary Aspects of Peasant Insurgency in Colonial India, Delhi, Oxford University Press, 1983; Gayatri Chakravorty Spivak, "Subaltern Studies: Deconstructing Historiography", en Ranajit Guha (ed.), Subaltern Studies IV: Writings on South Asian History and Society, Delhi, Oxford University Press, 1985, pp. 330-363, y Rosalind O'Hanlon, "Recovering the Subject: Subaltern Studies and Histories of Resistance in Colonial South Asia", Modern Asian Studies, vol. 22, núm. 1, 1988, pp. 189-224. 
En este escenario más amplio, mientras realizaba los estudios del programa (de honores académicos) de licenciatura en historia en St. Stephen's College, varios de mis compañeros y yo tuvimos atisbos de la excitación intelectual que rodeaba la aparición de los estudios subalternos. ${ }^{8}$ Pronto, cuando cursaba una maestría (regular) en historia (moderna), también en la Universidad de Delhi, los debates y su fermento llevaron a involucramientos críticos más amplios con corrientes historiográficas y teóricas en desarrollo. Ahí, mientras los estudios subalternos apuntaron poderosamente en direcciones históricas más nuevas, también pareció que el emprendimiento privilegiaba los momentos espectaculares de las rebeliones abiertas de los subalternos sobre las negociaciones de poder más rutinarias y cotidianas de estos grupos. Esto sugirió expresiones inadecuadas y abreviadas de cultura y conciencia, de religión y casta, dentro del proyecto. ${ }^{9}$

No es de sorprender que cuando buscaba un tema de investigación para la maestría en historia, también en la Universidad de Delhi, me interesara la conducta de la resistencia en un lenguaje religioso. Específicamente, quería rescatar tales negociaciones y refutaciones a la autoridad de su estado de subordinación -como insustanciales, incluso epifenomenales-a las determinaciones subyacentes de imperativos interminablemente económicos o de política principalmente progresiva, que abundaba en las historias heroicas de la época. Más bien, estaba en juego la manera en que las instituciones y las imaginaciones de la casta, las prácticas y los procesos de la religión

${ }^{8}$ La historia de cómo, a finales de la década de 1970, un pequeño grupo de entusiastas historiadores del sur de Asia, muy jóvenes y la mayoría en Inglaterra, sostuvo reuniones con el distinguido académico senior de la India colonial, Ranajit Guha, quien impartía cátedra de historia en la Universidad de Sussex, para discutir a fondo una nueva agenda para la escritura de la historia - una que reconociera la centralidad de los grupos subordinados en el pasado y el presente-fue inspiradora por sí misma. Cuento esta historia y discuto la elaboración del proyecto de los estudios subalternos, que viera la publicación de 12 volúmenes editados y numerosas monografías por miembros del colectivo, en Dube, Stitches on Time, op. cit., pp. 129-163. Véase también Dube, "Critical Crossovers", op. cit., para consultar una exploración crítica más amplia del emprendimiento de los estudios subalternos.

${ }_{9}$ Considérese el breve ensayo que escribí cuando era un impetuoso joven: Saurabh Dube, "Peasant Insurgency and Peasant Consciousness", Economic and Political Weekly, vol. 20, núm. 11, 1985, pp. 445-448. 
podían estructurar críticamente y dar forma a las acciones y las expresiones de las comunidades subordinadas. En cuanto al tema de estudio, por suerte encontré una casta-secta hereje e "intocable", los satnamis de Chhattisgarh. Los auspicios de mis padres demostraron ser importantes aquí, pues muchos años atrás ambos habían realizado su investigación de doctorado, tanto en términos lingüísticos como culturales, en esta gran región de India central.

Mientras trabajaba en una historia social de los satnamis para mi tesis de maestría, las potencialidades y los problemas de los estudios subalternos relacionados con las temporalidades se colocaron en primer plano, de maneras irresolutas pero insistentes. Por un lado, los análisis dentro del emprendimiento ubicaron las acciones y las aprehensiones de estos grupos como enteramente contemporáneas y formativamente coetáneas con el tiempo-espacio de la colonia británica y la nación india. Así, en sus escritos sobre el insurgente campesino en la India del siglo XIX, especialmente a través de su crítica de la noción de lo "prepolítico", Ranajit Guha interpretó proféticamente a este sujeto histórico como completamente contemporáneo de los procesos de colonialismo y nacionalismo y como su constituyente. ${ }^{10}$ Esto sirvió para analizar esquemas temporales comunes, dominantes y únicos, pero a la vez jerárquicos, que mostraron al subalterno no alfabetizado como rezagado del tiempo de lo moderno. ${ }^{11}$ Por otro lado, las sensibilidades de un paternalismo recuperativo indicaron que, en el proyecto, los significados y las motivaciones de estos pueblos se tamizaron simultáneamente a través de la distinción maestra entre comunidad y Estado. Podría decirse que esto reinstauraría de manera ambivalente los diseños temporales exactos y exclusivos, pero temporales, que los estudios subalternos habían deshecho. Ahora parecía que los

${ }^{10}$ Guha, Elementary Aspects..., op. cit. Dipesh Chakrabarty desarrolló más adelante tal lectura de la obra de Guha sobre el campesino insurgente en la India colonial, aunque no dedica el tiempo suficiente a las tensiones presentes en Elementary Aspects, así como en otros escritos de Guha. Dipesh Chakrabarty, Habitations of Modernity: Essays in the Wake of Subaltern Studies, Chicago, University of Chicago Press, 2002, pp. 8-14.

${ }^{11}$ No es posible exagerar la importancia del trabajo de Guha. Brindo una discusión sobre su prominencia, así como de los principales argumentos de Elementary Aspects, en Dube, Stitches on Time, op. cit., pp. 143-53. Véase también Dube, "Peasant Insurgency and Peasant Consciousness", op. cit. 
subalternos habitaban un tiempo anterior/a priori definido, que dependía de una tradición implícitamente inmutable, marcada por un espacio pasivo y moldeada por la mano muerta de la cultura gobernante. Así, fue sólo después de que estos grupos subordinados se embarcaran en la insurgencia, marcando una ruptura con su condición usual, cuando emergieron dentro del escenario temporal de la política moderna y, en realidad, a la vanguardia del mismo. ${ }^{12}$

Desde luego, no experimenté ni expresé las cosas de esta forma, pero las insinuaciones de la incertidumbre estaban al acecho, como una presencia un tanto sombría. De hecho, lejos de ser incapacitante, la ambigüedad fue productiva. Como señal de los tiempos, la tensión fue fructífera. Ahora, junto con otras tendencias teóricas, retomé críticamente los estudios subalternos para desarrollar sus primeras sensibilidades, que colocaban a los protagonistas desposeídos en el interior de la historia de manera formativa, a la vez que investigué sus últimos énfasis, que presentaron a estos sujetos como inciertamente fuera de tiempo.

Así, buscando entender las expresiones de los satnamis sobre el pasado que se centraron en sus gurús/preceptores, encontré en los mitos del grupo una modalidad de conciencia histórica que elaboró convenciones definidas. Aquí se encontrarían interpretaciones y procedimientos que accedían y excedían las configuraciones brahmán-reales y popular-devocionales, pero también las representaciones imperiales y nacionalistas, expresando temporalidades que se superponían. Sencillamente, las concepciones satnami del pasado eran enteramente contemporáneas de la historiografía moderna, e incluso reflejaban sus presunciones más que señalar una excepción exótica dictada por los imperativos de una temporalidad jerárquica pero singular. ${ }^{13}$ Asimismo, al centrar la atención en la justicia colonial y

${ }^{12}$ De acuerdo con Guha, la insurgencia era la "esencia" de los emprendimientos subalternos. Se trató de un momento expresivo enteramente autónomo y verdaderamente emancipatorio que involucró una "reversa prescriptiva" que tenía por objetivo la completa subversión y borramiento de la insignia de subalternidad. Guha, Elementary Aspects..., op. cit., passim.

${ }^{13}$ Saurabh Dube, "Social History of Satnamis of Chhattigarh", tesis de maestría inédita, Delhi, University of Delhi, 1988; gran parte de este trabajo se materializó después en Saurabh Dube, "Myths, Symbols, Community”, en Partha Chatterjee y 
las disputas aldeanas en la región de Chhattisgarh, lo que pasó a primer plano fueron las conversaciones contenciosas, las imbricaciones mutuas y los enfrentamientos formativos entre la ley/el orden moderno y las legalidades/ilegalidades populares. Es decir, lejos de la indolente oposición entre los procesos (tradicionales) de disputa de lo folclórico y las reglas occidentales-adjudicatorias (modernas), que temporal y espacialmente segregan estos terrenos en dominios previos y posteriores, estaban en juego incesantes embrollos entre las normas cotidianas, los deseos familiares y las patologías ajenas, lo que una vez más señalaba temporalidades enredadas. ${ }^{14}$

De hecho, en retrospectiva, estaba explorando procesos que trenzaban el tiempo, el espacio y sus engranajes. Sin embargo, en ese momento las preocupaciones se centraban, por ejemplo, en la antinomia absoluta, incluso aritmética, entre la élite y lo subalterno. Ahora, leída a través de los filtros de los patricios y los plebeyos en la Inglaterra del siglo XVIII o de los contornos de conciencia de los esclavos afroamericanos en el sur de Estados Unidos, ${ }^{15}$ esta oposición a los estudios subalternos englobaba la creación de los subalternos y las élites - de hecho, de la clase, la comunidad y el género- como procesos relacionales, o hacía cortocircuito con ellos. Además, aquí parecía haber una vacilación entre el privilegio de los códigos elementales, o estructuras subyacentes, que gobernaban la acción/insurgencia subalterna por un lado, y una celebración un tanto ingenua de su capacidad agentiva/autonomía ingobernada, por el otro. Abordada mediante debates sobre la relación entre la capacidad agentiva y la estructura, especialmente como se expresa en la obra de Philip Abram, Pierre Bordieu y Anthony Giddens, tal fluctuación parecía ser analíticamente inade-

Gyanendra Pandey (eds.), Subaltern Studies VII. Writings on South Asian History and Society, Delhi, Oxford University Press, 1993, pp. 121-156.

${ }_{14}$ Dube, "Social History", op. cit.; Saurabh Dube, "Telling Tales and Trying Truths: Transgressions, Entitlements and Legalities in Village Disputes, Late Colonial Central India”, Studies in History, vol. 13, núm. 2, 1996, pp. 171-201.

${ }^{15}$ E. P. Thompson, Customs in Common, Nueva York, New Press, 1991; Lawrence Levine, Black Culture and Consciousness: Afro-American Folk Thought from Slavery to Freedom, Oxford, Oxford University Press, 1977; Eugene Genovese, Roll, Jordan, Roll: The World the Slaves Made, Nueva York, Pantheon Books, 1978. 
cuada, persistentemente problemática y frecuentemente improductiva. ${ }^{16}$ Aun así, mi argumento es que estas oposiciones fáciles y estos análisis ambivalentes conllevaban implicaciones aún más amplias. Aunque apenas se expresa de esta manera, fue difícil no sentir un desasosiego persistente y latente ante las determinaciones inquietantes del tiempo singular y jerárquico que indicaban, además, espacios sociales antinómicos dentro de los estudios subalternos. ${ }^{17}$

Claramente, mi proyecto de investigación - y mis intereses académicos en general- se basaba en la interacción entre historia y antropología. Por ello, me convertí en un ávido lector del campo emergente de la antropología histórica, particularmente de obras que analizaban la historicidad y la temporalidad, la práctica y el proceso, el significado y el poder en África y en Oceanía, en Europa y en América. Ahora quedaba claro que, aunque la especialización de la antropología india a partir de la década de 1950 fue apuntalada con demarcaciones disciplinarias claras con la historia, exactamente en este escenario hubo esfuerzos discretos de algunos antropólogos por abordar temas históricos. Al mismo tiempo, también era evidente que tales esfuerzos se preocupaban menos por repensar la antropología y la historia desdibujando las fronteras disciplinarias que por expresar consideraciones antropológicas convencionales, basándose entonces en materiales históricos (muchos de los cuales siguieron siendo sospechosos para los historiadores profesionales de la época). Además, hasta bien entrada la década de 1960, estos esfuerzos se vieron influidos con frecuencia por formulaciones más amplias de las interacciones entre las "grandes" y las "pequeñas" tradiciones, entre procesos de "universalización” y "parroquialización”. ${ }^{18}$ Sostenidas por esquemas casi evolucionistas, estas proyecciones de una civilización india

${ }^{16}$ Anthony Giddens, Central Problems in Social Theory: Action, Structure, and Contradiction in Social Analysis, Berkeley, University of California Press, 1979; Bourdieu, Outline of a Theory of Practice, op. cit.; Philip Abrams, Historical Sociology, Shepton Mallet, Open Books, 1982.

${ }^{17}$ En esa época, el espacio era una idea un tanto nebulosa y brumosa para mí, excepto lo que de él se expresaba en Bourdieu, Outline of a Theory of Practice, op. cit.

${ }^{18}$ Robert Redfield, Peasant, Society, and Culture, Chicago, University of Chicago Press, 1956. 
global deshistorizaron precariamente el pasado y el presente, de manera que el tiempo y el espacio dependían el uno del otro de manera fácil e indolente. ${ }^{19}$

Al mismo tiempo, me di cuenta de que la institucionalización de la historiografía profesional del subcontinente había nada menos que procedido a distancia de la investigación antropológica durante la mayor parte del siglo XVIII. ${ }^{20} \mathrm{Al}$ mismo tiempo, desde mediados de la década de 1960, con influencia de corrientes divergentes del marxismo en el contexto de los levantamientos radicales en todo el mundo, las ciencias sociales atestiguaron una preocupación más generalizada por el lugar del campesinado en el desarrollo económico, el cambio histórico y la transformación revolucionaria. ${ }^{21}$ Estas preocupaciones ejercieron un efecto sobre la historiografía relativa a la sociedad campesina que implicó generalmente cuestiones de historia económica, pero también temas de cultura y poder. Dicho impacto se extendió a las historias sociopolíticas de los movimientos contracoloniales y de los nacionalismos populares de las agrupaciones campesinas, las clases trabajadoras y las comunidades indígenas. ${ }^{22}$ Todo ello preparó el escenario para debates críticos en la historia a partir de finales de la década de 1970 que reestructuraron la disciplina, particularmente al plantear nuevas preguntas e iniciar posibles conversaciones, por

${ }^{19}$ Para una discusión más amplia sobre la gama de este trabajo, incluidas las desviaciones, véase Saurabh Dube, "Introduction: Anthropology, History, Historical Anthropology", en Saurabh Dube (ed.), Historical Anthropology, Nueva Delhi, Oxford University Press, 2007, pp. 1-73.

${ }^{20}$ Para consultar una encuesta, véase Sumit Sarkar, Writing Social History, Delhi, Oxford University Press, 1997, cap. 1; véase Partha Chatterjee, "Introduction: History and the Present", en Partha Chatterjee y Anjan Ghosh (eds.), History and the Present, Delhi, Permanent Black, 2002, pp. 1-23.

${ }^{21}$ De hecho, los científicos sociales podían recurrir al registro histórico para explicar consideraciones de las sociedades y los movimientos campesinos. Véase, por ejemplo, Kathleen Gough, Rural Society in Southeast India, Cambridge, Cambridge University Press, 1981; D. N. Dhanagre, Peasant Movements in India, 1920-1950, Delhi, Oxford University Press, 1983; y Hetukar Jha, Social Structures of Indian Villages: A Study of Rural Bihar, Nueva Delhi, Sage, 1991.

${ }_{22}$ Por ejemplo, Ravinder Kumar (ed.), Essays on Gandhian Politics: The Rowlatt Satyagraha of 1919, Oxford, Clarendon Press, 1971; Gyanendra Pandey, The Ascendancy of the Congress in Uttar Pradesh, 1926-1934: A Study in Imperfect Mobilization, Oxford, Clarendon Press, 1978; David Hardiman, Peasant Nationalists of Gujarat: Kheda District 1917-1934, Delhi, Oxford University Press, 1981; y Majid Siddiqi, Agrarian Unrest in North India: The United Provinces, 1918-1922, Nueva Delhi, Vikas, 1978. 
ejemplo con la teoría crítica, los entendimientos sociológicos y la investigación etnográfica, aumentando con ello el estudio del sur de Asia. ${ }^{23} \mathrm{Al}$ mismo tiempo, las expresiones del tiempo y el espacio en las tendencias más nuevas llegaron con sus propios giros y propensiones, como se dijo anteriormente en el caso de los estudios subalternos.

\section{Involucramientos de aprendiz}

No es de sorprender que para mi doctorado en la Universidad de Cambridge, y el libro que surgió luego, buscara un diálogo entre los estudios subalternos, la antropología histórica y lo "cotidiano" como una perspectiva crítica, mientras continué mi investigación sobre los satnamis. ${ }^{24}$ Ahora, varios encuentros críticos y enredos contingentes -en el archivo, en el campo, en la biblioteca y en otros sitios- me señalaron el inmenso poder codificado en los signos y los símbolos, en las metáforas y los mapeos, y en las prácticas y las creencias del gobierno y del Estado. Tales formaciones de autoridad estructuraron de manera crucial imaginaciones y emprendimientos de lo subalterno y la comunidad. Estas insinuaciones iban en contra de la distinción innata entre la comunidad subalterna y el Estado moderno y tenían profundas implicaciones temporales-espaciales, que en diversos sentidos caracterizaron los estudios subalternos. Dos ejemplos importantes e ilustrativos deberían bastar.

El destacado estudio de Dipesh Chakrabarty sobre los trabajadores de los molinos de yute en el este de India hizo un llamado a la comprensión crítica de la experiencia diaria de las

${ }^{23}$ Bernard Cohn, "The Command of Language and the Language of Command", en Guha (ed.), Subaltern Studies IV, op. cit., pp. 276-329; Veena Das, "Subaltern as Perspective”, en Ranajit Guha (ed.), Subaltern Studies v: Writings on South Asian History and Society, Delhi, Oxford University Press, 1989, pp. 310-324; Upendra Baxi, “The State's Emissary': The Place of Law in Subaltern Studies”, en Chatterjee y Pandey (eds.), Subaltern Studies VII, op. cit., pp. 257-264. Véase también Sherry Ortner, "Resistance and the Problem of Ethnographic Refusal", Comparative Studies in Society and History, vol. 37, núm. 1, 1995, pp. 173-193.

${ }^{24}$ Saurabh Dube, "Religion, Identity, and Authority among the Satnamis in Colonial Central India", tesis doctoral inédita, Cambridge, University of Cambridge; Saurabh Dube, Untouchable Pasts. Religion, Identity, and Power among a Central Indian Community, 1780-1950, Delhi, Vistaar Publications, 1998. 
relaciones jerárquicas para atender a formas de cultura y conciencia que fueron "las 'olvidadas' del marxismo indio". Ésta fue la cuestión central para la historiografía de la clase trabajadora de la sociedad del sur de Asia, donde no se aplicaban las suposiciones de una cultura burguesa hegemónica. Sin embargo, Chakrabarty terminó por explorar al mismo tiempo la cultura y la conciencia de los trabajadores de las fábricas de yute de Calcuta como caracterizadas por atributos innatos que se asumieron a priori, a saber: "fuertes lealtades primordiales de comunidad, lengua, religión, casta y parentesco", que se interpretaron como características principalmente homeostáticas de una sociedad precapitalista. ${ }^{25}$ Podría decirse que una importante invitación a leer la diferencia en diseños dominantes de labor y capital, del pasado y el presente, fue socavada por el retorno a un plano maestro que dependía de una temporalidad singular que Chakrabarty se había propuesto cuestionar.

En un sentido relacionado, la crítica sostenida que hace Gyanendra Pandey de la construcción de la sociología colonial del "comunalismo" se apoderó de la comunidad —definida simplemente como la "sociedad india más allá de los confines del Estado”- como signo de alteridad y diferencia. Este signo sirvió para analizar el(los) conocimiento(s) dominante(s) de la colonia, la nación, el Estado y la historia. ${ }^{26}$ Aquí, precisamente separando a ambas, la presencia de la diferencia/comunidad se entendió como una oposición a las formaciones de poder/Esta-

${ }^{25}$ Nada de esto pretende negar la inmensa importancia de la monografía de Chakrabarty en el planteamiento de preguntas críticas sobre las clases trabajadoras, los subalternos y el capital para las ciencias sociales en general. Dipesh Chakrabarty, Rethinking Working-Class History: Bengal 1890-1940, Princeton, Princeton University Press, 1989, pp. xii, 69 y passim.

${ }^{26} \mathrm{La}$ importancia del estudio de Pandey radicó en que brindaba una perspectiva diferente y fresca en el ámbito más amplio de los estudios subalternos sobre el tema del comunalismo, bastante trillado. Gyanendra Pandey, The Construction of Communalism in Colonial North India, Delhi, Oxford University Press, 1990, p. 109 y passim. Desde una perspectiva diferente, Sandra Freitag examinó el dominio de las "arenas públicas" - un ámbito coherente y constante de comportamiento simbólico en el que la "comunidad se ha expresado y redefinido a través de actividades colectivas en espacios públicos”- como opuestas al (y, por ende, en su constitución interna, aisladas del) Estado imperial y sus instituciones. Sandria B. Freitag, Collective Action and Community: Public Arenas and the Emergence of Communalism in North India, Berkeley, University of California Press, 1989, p. 6 y passim. 
do. Esto sirvió para reprochar y sostener inciertamente una temporalidad histórica exclusiva, exactamente a través de expresiones agudas de espacios segregados de comunidad/diferencia y Estado/poder, donde las primeras asumieron una prioridad epistemológica y ética sobre los segundos.

Contra la veta de énfasis tan influyentes, mi propia obra sobre los satnamis dio seguimiento a los enredos entre comunidad/subalterno/diferencia y Estado/dominante/poder, cuando menos en cuatro formas superpuestas que expreso de manera un tanto telegráfica en el presente trabajo. ${ }^{27}$ Primero, la creación misma del emprendimiento con la casta-secta satnami fue moldeada por las dinámicas engranadas de significado y poder que se mencionaron anteriormente. Dichas dinámicas pusieron de manifiesto y analizaron el entretejido de las jerarquías divinas, rituales, sociales y gubernamentales, así como sus matrices temporales y espaciales concomitantes. Segundo, estaban en juego las formas en que los patrones de autoridad, dentro de los arreglos de casta, implicaron un trenzado formativo de protocolos de poder que eran a la vez sustantivos y simbólicos. Estos ejes enredados giraban en torno a la pureza y la contaminación rituales, la realeza cultural y las castas dominantes, y la gobernanza y la ley coloniales. Tercero, las concepciones históricas de los satnamis - materializadas en sus representaciones míticas y de otros órdenes- llegaron a espacios definidos de secta/casta y temporalidades noveles de orden/legalidad. Lo lograron negociando e investigando figuras de dominio que orquestaban los mundos “cósmico" y "social”, comúnmente engranados. En cuarto y último lugar, estas orientaciones hacia la autoridad y la alteridad encontraron expresiones diferentes, pero superpuestas en las organizaciones satnamis vinculadas al nacionalismo indio, sobre todo cuando tamicé las presunciones de la clase media a través de imaginarios subalternos en estos

${ }^{27}$ El final de los años 80 y el inicio de los 90 fue una época emocionante para realizar investigación en Cambridge, y mi propio trabajo retomó las preocupaciones surgidas de los escritos de Bayly y se benefició de conversaciones con Ajay Skaria sobre el trabajo de archivo y de campo, la historia y la antropología. C. A. Bayly, Indian Society and the Making of the British Empire, Cambridge, Cambridge University Press, 1987. La obra de Skaria se publicó después como Hybrid Histories: Forests, Frontiers and Wildness in Western India, Nueva Delhi, Oxford University Press, 1999. 
ámbitos, revelando con eso destellos alternativos de legalidad y legitimidad, de política y nación(es). ${ }^{28}$

Así, en el corazón de mi investigación yacía la interpenetración incesante entre aspectos constitutivos del poder estatal/gubernamental y las formas cotidianas de vida subalter$\mathrm{na} /$ comunitaria. Estos engranajes tocaban simultáneamente y revolvían una temporalidad singular y jerárquica y sus espacios antinómicos relacionados. Fueron exactamente estos enredos los que, sobre todo, se mantuvieron a distancia en la antropología y la historia del sur de Asia, como puede verse en importantes obras sobre el subcontinente. ${ }^{29}$ Se trataba de procedimientos generalizados de la espacialización del tiempo y la temporalización del espacio que sirvieron para escindir al subalterno y al Estado, a la comunidad y la historia, la tradición y la modernidad y la emoción y la razón como elementos que encarnan espacios separados mediante la suposición de una temporalidad exclusiva. Al mismo tiempo, una vez más, nada de eso apareció ante mí como una revelación centellante, sino que estas insinuaciones se desarrollaron poco a poco, pedazo a pedazo.

En este sentido, un proyecto sobre enredos evangélicos en la India central desempeña un papel central. Éste había encontrado sus primeras insinuaciones en la época de mi doctorado,

\footnotetext{
${ }^{28}$ Dube, "Religion, Identity, and Authority", op. cit.; Dube, Untouchable Pasts, op. cit.

${ }^{29}$ No pretendo con esto reivindicar un estatus exclusivo para mi obra ni negar contribuciones destacadas - entre las que se incluyen, sobre todo, aquellas provenientes del seno de los estudios subalternos- que desenredaron estos nudos de diversas maneras. Por ejemplo, pienso en la obra magistral de Shahid Amin sobre el nacionalismo subalterno y la nación monumental en el norte de India, y en la imaginativa digresión de Ajay Skaria hacia formas de salvajismo y conciencia histórica en la India occidental. Por un lado, todo es testimonio de la heterogeneidad formativa del proyecto de los estudios subalternos, y, por el otro, incluso estos estudios no pudieron darse cuenta por completo de sus propias implicaciones críticas: lucharon contra límites previos y poderosos binarios, pero no pudieron deshacerse por completo de su larga sombra. Shahid Amin, Event, Metaphor, Memory: Chauri Chaura 1922-1992, Berkeley, University of California Press, 1996; Ajay Skaria, "Writing, Orality, and Power in The Dangs, Western India, 1800s-1920s", en Shahid Amin y Dipesh Chakrabarty (eds.), Subaltern Studies IX: Writings on South Asian History and Society, Delhi, Oxford University Press, 1996, pp. 13-58; Skaria, Hybrid Histories, op. cit.; e Ishita Banerjee-Dube, “Taming Traditions: Legalities and Histories in Eastern India”, en Gautam Bhadra, Gyan Prakash y Susie Tharu (eds.), Subaltern Studies X: Writings on South Asian History and Society, Nueva Delhi, Oxford University Press, 1999, pp. 98-125.
} 
cuando llevé a cabo trabajo de archivo en registros de misioneros en Estados Unidos, pero se convirtió en mi primera investigación posdoctoral un mes después de haber sometido mi tesis doctoral. Se trata de un estudio sobre los misioneros evangélicos estadounidenses y los cristianos conversos "nativos" en la India colonial y la independiente. Combinando investigación de archivo y de campo, perspectivas etnográficas e históricas -que se conjugan aún más con consideraciones de teoría social-, el objeto de este proyecto ha sido al menos triple.

Primero, discute el entrelazamiento de los esfuerzos evangélicos y las prácticas de los conversos con formación de castasecta y la dinámica de la vida aldeana. Los engranajes contenciosos dieron forma a la empresa misionera y a un cristianismo vernáculo. En segundo lugar, considera las conjunciones y las contradicciones entre el proyecto misionero y el poder imperial, entre las iniciativas evangélicas y las congregaciones "de casa”, y entre un cristianismo vernáculo y las culturas coloniales. Estos vínculos tan cargados subyacían a las expresiones críticas de la modernidad, el evangelismo y el imperio. En tercer y último lugar, el estudio explora variadas expresiones de comunidad y nación tras la conversión. Éstas insinúan temas controvertidos de la "mayoría” y la "minoría”, de política y religión, y del ciudadano y el converso, especialmente en la India independiente. Todos estos procesos parecen moldeados por distinciones de género y casta y de raza y comunidad. ${ }^{30}$

Si bien así se desarrolló el estudio a lo largo de las últimas dos décadas, también sucede que desde un inicio mis preocupaciones eran disonantes con buena parte del estudio académico sobre el sur de Asia, incluidas en especial las demarcaciones incómodas del tiempo y el espacio en los estudios subalternos. Considérense ahora los acentos de mi proyecto en relación con los agudos engranajes entre misionero y converso, entre colonizador y colonizado, entre dominante y subalterno, entre cul-

${ }^{30}$ Para consultar parte de mi obra sobre el tema que articula estos énfasis, véase Dube, Stitches on Time, op. cit., y Dube, After Conversion: Cultural Histories of Modern India, Nueva Delhi, Yoda Press, 2010. El proyecto en términos más amplios se materializa en Saurabh Dube, Formations of an Evangelical Modernity: Christianity, Conversion, Colonialism 1860-2005, manuscrito, s.f.; y Saurabh Dube, Native Witness: Colonial Writings of a Vernacular Christianity, manuscrito, s.f. 
turas coloniales y cristianismo vernáculo, entre imperio y modernidad, y entre poder y diferencia, que se apuntalaron con expresiones superpuestas, pero heterogéneas, del tiempo y el espacio. En contraste, el trabajo de los estudios subalternos se basaba en su mayor parte en mantener al colonizador y al colonizado, al dominante y al subalterno, el imperio y la modernidad, y el poder y la diferencia separados uno del otro, con lo cual se ponían sobre la mesa demarcaciones temporoespaciales clave. Esto debería quedar claro a partir de los siguientes ejemplos.

Hemos notado que Ranajit Guha (y los estudios subalternos en general) presentaba al insurgente subalterno como constituyentemente contemporáneo del colonialismo británico y el nacionalismo indio en el subcontinente. Aunque estaba socavada simultáneamente por demarcaciones temporoespaciales del campesino del sur de Asia, la medida analítica insinuaba las posibilidades de abordar al subalterno en la India imperial (y la independiente) como sujeto de la modernidad, y por lo tanto de entender la modernidad misma de nuevas maneras, ${ }^{31}$ pero esto no llegó a suceder. Apenas unos años después, Guha se pronunció en favor de la "dominancia sin hegemonía” en la India colonial, planteando un arquetipo de hegemonía burguesa donde la persuasión supera a la coerción en la composición de su dominancia. ${ }^{32}$ Se planteaba el prototipo clásico del Estado liberal hegemónico que representaba a una burguesía revolucionaria y política democrática en la Gran Bretaña metropolitana, contra lo cual se erguía el malhadado ejemplo de la dominancia sin hegemonía en la India colonial.

Moldeado por suposiciones inmaculadas de una vigorosa democracia y una política liberal vital del Occidente moderno, el análisis de Guha interpretó la narrativa histórica central del poder en el subcontinente bajo el gobierno colonial como fracasada y deficiente. ${ }^{33}$ Evacuados de su propia particularidad,

${ }^{31}$ Sobre la idea de "sujetos de la modernidad" en relación con "el sujeto moderno”, véase Dube, Stitches on Time, op. cit.; Dube, After Conversion, op. cit., y Dube, Subjects of Modernity, op. cit.

${ }^{32}$ Ranajit Guha, Dominance without Hegemony: History and Power in Colonial India, Cambridge, Harvard University Press, 1997, cap. 1.

${ }^{33} \mathrm{La}$ carencia se expresó diligentemente por la mala fe de un poder imperial autocrático y los límites arraigados de una burguesía indígena inútil. Asimismo, a diferencia de muchos de sus colegas más jóvenes en el emprendimiento de los estudios 
los significados de estos pasados de dominancia en la colonia -en contraste con los de la insurgencia subalterna- fueron inherentes en su permanente retraso respecto al tiempo y el espacio de Europa. En estas proyecciones teleológicas de los pasados coloniales y las historias metropolitanas, las transiciones incompletas de los primeros se miden constantemente contra las trayectorias zalameras de las últimas, de manera que se apuntalan unas a las otras. Están en juego expresiones de una exclusiva temporalidad jerárquica que segrega espacialmente a Gran Bretaña y a India, al imperio y su puesto, a Occidente y al Resto. ${ }^{34}$ En términos sencillos, todo esto fue contrario a mis intentos de explorar la constitución común y la labor recíproca de la modernidad y el colonialismo en la metrópolis y en las márgenes, así como las orientaciones insinuadas por tales acentos en lo temporal, lo espacial y sus entrelazamientos.

Lo anterior me lleva al segundo ejemplo. La influyente The Nation and its Fragments, de Partha Chatterjee, ubicó críticamente formas de comunidad en regímenes de modernidad en lugar de reificarlas como "remanentes premodernos que una distraída Ilustración olvidó borrar". ${ }^{35}$ (Este hecho suele pasarse por alto en críticas comunes y descuidadas del trabajo. $)^{36} \mathrm{El}$ movimiento hizo posible que Chatterjee interpretara lecturas vigorosas que analizan las categorías de Estado y sociedad civil, a la vez que le permitió sugerir otras imaginaciones de comunidad, nación(es) y modernidad. También es cierto que tales posibilidades en la obra de Chatterjee se sostienen y se socavan

subalternos que expresaron espacios escindidos de comunidad y Estado, de diferencia y disciplina, para Ranajit Guha la principal división se encontraba entre el subalterno insurgente y la élite dominante.

${ }^{34}$ En conjunto, es precisamente atendiendo a los discursos simultáneos en diferentes lenguas de los escritos de Guha como se hace posible cuestionar la noción de una brecha implacable, una contradicción innata, entre un régimen democrático moderno en casa y sus interminables omisiones retrógradas en la colonia; además, cuestionar afirmaciones sobre la naturaleza enteramente excepcional de la "gubernamentalidad colonial" y la "modernidad colonial"; y, finalmente, hacer esto para dar seguimiento a la constitución mutua y la labor recíproca de la modernidad y el colonialismo en la metrópolis y en las márgenes.

${ }^{35}$ Partha Chatterjee, The Nation and its Fragments: Colonial and Postcolonial Histories, Princeton, Princeton University Press, 1993.

${ }^{36}$ En lugar de ello, más allá de los estudios subalternos, la importancia del libro de Chatterjee radica en su crucial aportación a nuevos énfasis en los estudios críticos -especialmente en una revigorizada antropología cultural- de Estado y nación. 
por dos pautas: primera, la aguda separación que establece entre Estado y comunidad, que acota totalmente cualquier intercambio entre los símbolos del Estado y los contornos de la comunidad, y segunda, su notable aseveración de que "por su propia naturaleza, la idea de comunidad marca un límite al ámbito del poder disciplinario". ${ }^{37}$ Tomados en conjunto, en The Nation and its Fragments, los precisos resquicios de orientaciones más nuevas hacia la modernidad y la comunidad no pueden separarse de postulaciones de la obra sobre el potencial de la modernidad realizada mediante la virtud de la comunidad, que insinúa una diferencia pura, una alteridad inmaculada.

Aprender tanto de las posibilidades y los problemas de esta obra ha requerido al menos dos elementos. Por un lado, es imperativo prestar atención a la interrogación de Chatterjee de una modernidad exclusiva, centrada en el Estado y el capital, como una vía que agote los imaginarios y las acciones modernas. Esta crítica se lleva a cabo en nombre de la comunidad, pero tiene implicaciones mucho más amplias. Por otro lado, es crucial registrar que la afirmación de la obra de un único tiempo histórico de comunidad y Estado es principalmente un ardid narrativo, un marcador temporal de la modernidad política. Esto se llena con dos argumentos en competencia: el del capital y el de la comunidad. Resulta importante que, en este sentido, la comunidad/la diferencia se basen en una prioridad y una precedencia epistemológicas y éticas sobre el capital/el poder: en esencia, estas categorías opuestas insinúan esencias fuertemente separadas, locus espaciotemporales definidos, temas que retomaré más adelante.

\section{Enredos de maestro}

De hecho, varias de estas preocupaciones se aclararon poco a poco cuando me uní al profesorado del Centro de Estudios de Asia y África de El Colegio de México y me mudé a la Ciudad de México a mediados de la década de 1990. Además, se puso de manifiesto otro conjunto de preocupaciones superpuestas pero 
definidas. Pronto me percaté de que, en los mundos latinoamericanos, Asia y África se tamizaban a partir de perspectivas de espacio y tiempo bastante particulares y un tanto peculiares, tanto en los ámbitos cotidianos como en los académicos. Con la Latinoamérica (mestiza) dispuesta de manera incierta, pero fácil, a semejanza de un Occidente moderno reificado, la diferencia cultural/espacial y la otredad temporal/social de África y de Asia, actuando en tándem, significaban una marca de encantamiento, algo bello. Pero el "retraso" político-económico de estos continentes, que implicaba un retraso de tiempo, también encarnaba un impedimento histórico, una falta de modernidad, una inferioridad socioespacial temporal, algo feo. Desmenuzando estas distinciones espaciotemporales simultáneas, emprendí estudios sobre la colonialidad/decolonialidad del poder, así como una gama de vitales escritos sobre y desde el sur del río Bravo. De hecho, mientras trabajaba en una yuxtaposición y conexión de entendimientos críticos entre Latinoamérica y el sur de Asia, incluida sobre todo en la enseñanza, fueron la modernidad y sus múltiples vínculos con la Ilustración y el imperio, con la razón y la raza, y con las colonias (con pobladores y sin ellos) y las naciones, los que emergieron como ámbitos apropiados de conversación.

En Latinoamérica se han aprehendido cuestiones del colonialismo como ocupantes de un pasado oscuro y distante. Después de dos siglos de libertad formal, la modernidad se entiende como un atributo de la nación independiente, desconectada del imperio, que es un tiempo lejano, un extraño espacio, un episodio y una entidad casi olvidados, excepto entre los académicos especialistas. Al mismo tiempo, pronto se hizo igualmente evidente que, siguiendo una estética barroca, los pasados de lo cotidiano colonial con frecuencia también se presentan en estos terrenos en formas de celebración. Así, encantados admiradores nos han descrito Coyoacán - una colonia (vecindario) del siglo xvi al sur de la Ciudad de México-, donde hemos vivido 20 años, como un lugar "muy colonial". Dicho de otro modo, la presencia continua de marcadores temporoespaciales previos puede representar el triunfo de la historia, uniendo el pasado con el presente en la visualización de la nación y la civilización. 
Contra estas disposiciones dominantes, un importante corpus de pensamiento crítico sobre Latinoamérica se ha concentrado en los esquemas subterráneos, las presunciones generalizadas y las apariciones crispadas de lo moderno y lo colonial. ${ }^{38}$ Este corpus toma como punto de partida la "primera modernidad" del sur de Europa - unida por el Renacimiento, la conquista del "Nuevo Mundo" y los imperios de España y Portugal- en las márgenes y en la metrópolis. Asimismo, con ello considera críticamente el lugar y la presencia de estipulaciones coloniales de poder en provisiones modernas de conocimiento. Los escritos forjan su camino nada menos que a través de la "segunda modernidad" del Norte del mundo, constituida por los imperios de la Ilustración y posteriores. Esto refleja la modernidad como un proyecto profundamente ideológico y un aparato de dominio primario en el pasado, el presente y la posteridad. En conjunto, en estos entendimientos la posibilidad recursiva de la redención secular-mesiánica aparece con frecuencia como un horizonte futuro exclusivo. ${ }^{39}$ Ahora, estos énfasis han puesto en primer plano, de manera formidable, las propensiones eurocéntricas y la violencia

${ }^{38}$ No resulta sorprendente que poco después de mudarme a la Ciudad de México intentara iniciar un diálogo con dichos escritos como parte de conversaciones críticas entre distintas perspectivas desde/sobre el sur. Véase, por ejemplo, Saurabh Dube, Ishita Banerjee-Dube y Edgardo Lander (eds.), Critical Conjunctions: Foundations of Colony and Formations of Modernity, Durham, Duke University Press, 2002, pp. 195422; Saurabh Dube, Ishita Banerjee-Dube y Walter Mignolo (eds.), Modernidades coloniales: Otros pasados, historias presentes, México, El Colegio de México, 2004; Saurabh Dube e Ishita Banerjee-Dube, Unbecoming Modern: Colonialism, Modernity, Colonial Modernities, Nueva Delhi, Social Science Press, 2006.

${ }^{39}$ Este corpus es inmenso, antes conocido bajo la rúbrica de (entendimientos de la) "colonialidad del poder" y más recientemente agrupado bajo (perspectivas sobre) la "decolonialidad del conocimiento". Con vínculos a tradiciones previas en Latinoamérica de la teoría de la "dependencia” y la "teología de la liberación”, en sus configuraciones actuales las obras de Aníbal Quijano, Enrique Dussel y Walter Mignolo, así como un grupo de académicos más jóvenes, algunos de los cuales se citarán más adelante, han sido una importante presencia. Si bien sería un error minimizar sus distinciones internas plegando rápidamente estos escritos entre sí, también es cierto que las lecturas en estas perspectivas con frecuencia intentan expresar sus principales puntos en común, su unidad mutua, más que detenerse en sus diferencias. Véase, por ejemplo, Ramón Grosfoguel, "Decolonizing Post-colonial Studies and Paradigms of Political Economy: Transmodernity, Decolonial Thinking, and Global Coloniality”, Transmodernity: Journal of Peripheral Cultural Production of the Luso-Hispanic World, vol. 1, núm. 1, 2011, pp. 1-37. La mayoría de los artículos sobre la colonialidad/decolonialidad de esta revista, Transmodernity, corroboran mis afirmaciones. 
epistémica de la modernidad, que se entiende como ya/siempre colonial. Además, han subrayado la importancia de otras formas de gnosis y conocimientos que revelan horizontes distintos a los de lo moderno dominante occidental. ${ }^{40}$ Tales insinuaciones conllevan muchos elementos de valor. Al mismo tiempo, el desentrañamiento de la "colonialidad del poder" y la "decolonialidad del conocimiento" por parte de estos escritos se basa en presunciones de la naturaleza esencialmente distópica de la primera y las posibilidades innatamente utópicas en términos éticos del segundo. ${ }^{41}$ Tales afirmaciones no sólo insinúan una tendencia a priori, sino que lo hacen al tiempo que comportan implicaciones profundamente temporales y espaciales. Basaré mi discusión en los argumentos y las implicaciones del filósofo argentino Enrique Dussel para explorar los énfasis de la perspectiva de la colonialidad/decolonialidad, especialmente los que dependen del espacio y el tiempo.

Para Dussel son cruciales los escritos del filósofo Emmanuel Levinas sobre ética, alteridad y exterioridad. ${ }^{42}$ Para Levinas, como saben los iniciados, el "otro" es una presencia constitutiva inquietante que relacionalmente revela los límites y los horizontes del "ser", de manera que la "ética [fue] la primera filosofía”,

${ }^{40}$ Enrique Dussel, The Invention of the Americas: Eclipse of "the Other" and the Myth of Modernity, Nueva York, Continuum, 1995; Enrique Dussel, "Europe, Eurocentrism and Modernity (Introduction to the Frankfurt Lectures)", Boundary 2, vol. 20, núm. 3, 1993, pp. 65-76; Walter Mignolo, The Darker Side of Renaissance: Literacy, Territoriality, and Colonization, Ann Arbor, University of Michigan Press, 1995; Nelson Maldonado-Torres, Against War: Viezes from the Underside of Modernity, Durham, Duke University Press, 2008.

${ }^{41}$ Éstas son sensibilidades implícitas persistentes, a veces expresadas de manera impactantemente explícita, que recorren la mayoría de las expresiones de las perspectivas coloniales/decoloniales.

${ }^{42}$ Emmanuel Levinas, Time and the Other, tr. Richard A. Cohen, Pittsburgh, Duquesne University Press, 1987; Enrique Dussel, Ética de la liberación en la edad de la globalización y de la exclusión, Madrid, Trotta, 1998. Véase también Emmanuel Levinas, Totality and Infinity: An Essay on Exteriority, tr. Alphonso Lingis, Pittsburgh, Duquesne University Press, 1987. Al considerar la relación entre Levinas y Dussel, me pareció especialmente iluminador el texto de Silvana Rabinovich, "Alterity", en Robert McKee Irwin y Mónica Szurmuk (eds.), Dictionary of Latin American Cultural Studies, Gainesville, University Press of Florida, 2012, pp. 17-22; y Nelson MaldonadoTorres, Against War, op. cit. Maldonado-Torres atiende éticamente a las tensiones y las contradicciones, así como a los problemas y las posibilidades en la obra tanto de Levinas como de Dussel, sobre todo cuando se les lee juntas. Habiendo aprendido de su espléndida obra, también es cierto que la afirmación crítica que busco se interseca con los énfasis de Maldonado-Torres y también se separa de ellos. 
más que la epistemología o, digamos, la ontología heideggeriana del ser. ${ }^{43}$ Dussel transforma estos atributos innatamente emergentes, necesariamente no empíricos del encuentro "ético entre lo Igual y lo que permanece por siempre exterior a ello" en espacios escindidos y sustancializados. Aquí se encuentran espacios escindidos con referentes objetivos geopolíticos concretos, a saber: Europa y Latinoamérica. ${ }^{44}$ En este escenario resulta que Latinoamérica no sólo ya es temporalmente contemporánea de Europa/Euroamérica, lo que revela el lado oscuro de la última, sino también que, proyectada como un espacio unitario que subsume fácilmente también el ser del filósofo Dussel, se representa como siempre adelantada a Europa en términos éticos, lo que se totaliza en un espacio invariante de hegemonía no ética, y que expresa las dimensiones coloniales de poder moderno. Todo esto tiene amplias implicaciones.

Para empezar, no debe sorprender que la escisión inicial y singular de Dussel entre Europa y Latinoamérica -junto con el énfasis exclusivo sobre la "colonialidad del poder" - se revisara pronto en el seno del corpus más amplio de la decolonialidad. Se vio suplantada por el contraste geopolítico, espacial-moral, entre la hegemonía de Europa/Euroamérica y el "otro [o subalterno] lado de la diferencia colonial", la última nombrada en diversas formas: "transmodernidad", "conocimiento fronterizo" y "perspectivas decoloniales". ${ }^{45} \mathrm{Al}$ mismo tiempo, estas entidades segregadas éticamente han seguido representando, en un escenario histórico compartido, un drama de principios, un in-

${ }^{43}$ Levinas, Time and the Other, op. cit.; Maldonado-Torres, Against War, op. cit.

${ }^{44}$ Dussel, Ética de la liberación, op. cit.; Maldonado-Torres, Against War, op. cit., p. 176.

${ }^{45}$ Dussel, The Invention of the Americas, op. cit.; Enrique Dussel, "Transmodernity”, en Dube, Banerjee-Dube y Lander (eds.), Critical Conjunctions, op. cit., pp. 221-244; Aníbal Quijano, "Colonialidad y modernidad/racionalidad”, Perú Indígena, vol. 13, núm. 29, 1992, pp. 11-21; Aníbal Quijano, "La colonialidad del poder y la experiencia cultural latinoamericana”, en Roberto Briceño-León y Heinz R. Sonntag (eds.), Pueblo, época y desarrollo: la sociología de América Latina, Caracas, Nueva Sociedad, 1998, pp. 139-155; Aníbal Quijano, "Coloniality of Power, Ethnocentrism, and Latin America”, Nepantla, vol. 1, núm. 3, 2000, pp. 533-580; Walter Mignolo, Local Histories/Global Designs: Essays on the Coloniality of Power, Subaltern Knowledges and Border Thinking, Princeton, Princeton University Press, 2000; Grosfoguel, "Decolonizing Post-colonial Studies”, op. cit., y Walter Mignolo, The Darker Side of Western Modernity: Global Futures, Decolonial Options, Durham, Duke University Press, 2011. 
terminable choque entre lo malo y lo bueno, el mal y la virtud, la inmoralidad y la moralidad.

Además, las afirmaciones originales de Dussel tenían que ver con una sustitución de la fenomenología por una política orientada éticamente (recuérdese la proclamación de Levinas de la "ética como la primera filosofía"). Sin embargo, bajo el giro decolonial, la primacía de la ética y la política ha implicado que aparezcan elididas, implícitamente y a priori, con la epistemología y la ontología, la lectura/escritura y el ser/convertirse, como maneras de conocer y actuar. Aquí se encuentra un antídoto a la autoridad ante la distopía del poder. Dicho de otro modo, se considera que el "lado subalterno de la diferencia colonial" tiene precedencia de principios - que se entiende como siempre triunfante- sobre la "colonialidad del poder". Por lo tanto, no sólo se sostiene que los académicos decoloniales apoyan el otro lado de la diferencia colonial, sino que ya son lo mismo que portadores críticos de conocimiento(s) subyugados, todos habitantes comunes de las márgenes geopolíticas.

Por último, la lógica de tales espacios tan segregados en entendimientos decoloniales orquesta el tiempo y la temporalidad en formas determinadas. Lo temporal aparece aquí como un indicador cronológico que define la contemporaneidad innata de la modernidad/colonialidad y sus otros. Destaca que tal simultaneidad señale discretas verdades. En esta perspectiva, formas de colonialismo, modernidad y nación ponen de manifiesto cambios y transformaciones jurídico-políticos, pero la colonialidad misma del poder se plantea como poseedora de atributos innatamente inmutables. Asimismo, podría reconocerse que el otro lado de la colonialidad, incluidas perspectivas decoloniales, tiene manifestaciones heterogéneas, pero se asume que la lógica central de estas formaciones es inherente a incesantes interrogaciones de la modernidad/colonialidad, así como a expresiones heroicas de la pluriversalidad/diversalidad. Esto se debe a que se presume que las perspectivas decolonizadoras poseen una precedencia innata a priori - en términos de ética y política, de conocer y de ser- sobre el poder moderno. ${ }^{46}$

${ }^{46} \mathrm{Mi}$ preocupación aquí tiene que ver con las suposiciones y presunciones -frecuentemente tácitas y subexpresadas- que apuntalan estos escritos. Cabe enfatizar también que el tiempo puede representarse en este corpus como una categoría de cálculo 
En términos sencillos, me emocionaban los problemas planteados, pero me preocupaban las respuestas que ofrecía este formidable corpus. ${ }^{47}$ Mientras luchaba con los problemas y los argumentos que esbocé anteriormente -un proceso de incomodidad implícita más que de resolución fácil-, me di cuenta también de que mis lecturas sobre las perspectivas poscoloniales y los estudios subalternos estaban caracterizadas por una sensibilidad similar hacia el registro de sus dificultades y posibilidades simultáneas. ${ }^{48}$

Por un lado, los argumentos persistentes de lo poscolonial y lo subalterno como categorías y perspectivas registran una ambigüedad improductiva. De hecho, esta oscuridad poco útil se vincula íntimamente a las afirmaciones exclusivas que se hacen en nombre de estas formaciones de conocimiento. La aparente certidumbre y la ambivalencia real sobre las demarcaciones de tiempo y espacio desempeñan un papel importante en este sentido. Por lo tanto, como han enfatizado en repetidas ocasiones prominentes críticos poscoloniales, el concepto de lo poscolonial ha descansado en la división entre lo colonial y lo poscolonial. ${ }^{49}$ Aquí, una trayectoria temporal enteramente

\footnotetext{
y no de experiencia, atribuida a la cultura y no a la naturaleza. Ahora, el tiempo se expresa explícitamente como un concepto central del imaginario del sistema mundial colonial/moderno, enteramente entrelazado con la colonialidad del poder y la producción de diferencia colonial. Tal investigación del tiempo como colonización, como cálculo y representación, abre posibilidades críticas. Al mismo tiempo, sin embargo, tal entendimiento permanece circunscrito por su planteamiento de inconmensurables éticos/epistémicos/ontológicos entre la colonialidad/modernidad y la alteridad/ identidad, que se exploraron anteriormente. Así, busca encontrar totalmente otras expresiones del espacio/tiempo más que permanecer o analizar la heterogeneidad formativa del espacio y el tiempo, como práctica y producción, en mundos sociales en general, a través de los “continentes”. Véase, por ejemplo, Walter Mignolo, "Coloniality at Large", en Saurabh Dube (ed.), Enchantments of Modernity: Empire, Nation, Globalization, Londres, Routledge, 2009, pp. 67-95. Véase también Walter Mignolo, "The Enduring Enchantment (Or the Epistemic Privilege of Modernity and Where to Go from Here)", South Atlantic Quarterly, vol. 101, núm. 4 (número especial editado por Saurabh Dube), 2002, pp. 927-954.

${ }^{47}$ Parte de esta emoción y estas posibilidades se revelan en mi esfuerzo por entablar conversaciones con protagonistas de la colonialidad/decolonialidad, ya anotadas.

${ }^{48}$ Dube, Stitches on Time, op. cit.; Saurabh Dube, “Terms that Bind: Colony, Nation, Modernity", en Saurabh Dube (ed.), Postcolonial Passages: Contemporary History-Writing on India, Nueva Delhi, Oxford University Press, 2004, pp. 1-37.

${ }^{49}$ Anne McClintock, Imperial Leather: Race, Gender, and Sexuality in the Colonial Contest, Nueva York, Routledge, 1995; Ella Shohat, "Notes on the Post-colonial”, en
} 
exclusiva y espacios sociales formidablemente escindidos se sostienen mutuamente, de manera que los ardides narrativos del tiempo histórico llevan de un terreno totalizado (el colonial) a otro ámbito no diferenciado (el poscolonial). Lo anterior sirve para homogeneizar la diferencia crítica, instaurar la jerarquía histórica, elidir los espacios sociales desiguales y sanitizar la política poscolonial. ${ }^{50}$

Por otro lado, en diferentes disciplinas y diversos contextos académicos, los esfuerzos definidos que involucran y expresan las perspectivas poscoloniales y subalternas, pensadas en un sentido amplio, pueden leerse con cautela y entenderse que han llevado a cabo tareas sobresalientes. Para empezar, tales esfuerzos han repensando el imperio en diversas formas. En este sentido, han sido especialmente importantes los indicadores del juego previo y generalizado de los esquemas coloniales en los mundos contemporáneos. Estos énfasis han subrayado la enorme importancia y la influencia continua de los engranajes entre la Ilustración y el imperio, entre la raza y la razón, entre la metrópolis y las márgenes, y entre la religión y la política. Además, diversos escritos en este ámbito han cuestionado en varias ocasiones el lugar de un "Occidente" imaginario, pero palpable como historia, modernidad y destino, para todas las culturas y cada uno de los pueblos. Esto ha sugerido entendimientos más nuevos de comunidad, historia y modernidad, que han desafiado concepciones previas y modulares de estas categorías-entidades. Además, los esfuerzos que amplían las perspectivas subalternas y poscoloniales han desentrañado los términos y los límites del Estado, la nación y el ciudadano en mundos occidentales y no occidentales, con lo que subrayan

Padmini Mongia (ed.), Contemporary Postcolonial Theory: A Reader, Londres, A Hodder Arnold Publication, 1996, pp. 321-334.

${ }^{50}$ En realidad, esto no es todo. En el momento mismo en que los entendimientos poscoloniales hacen ver al colonizador y al colonizado como habitantes de la misma historia, deshaciendo las jerarquías temporales entre ellos, pueden segregar de manera implícita y nítida las habitaciones de Europa, su espacio-tiempo correcto, desde el de la colonia, al que se adjudica una exclusiva prioridad epistémica reveladora. Por lo tanto, vale la pena preguntar una vez más, como lo he hecho a lo largo de este ensayo, si no es importante permanecer con los límites constitutivos y las posibilidades formativas que apuntalan la heterogeneidad y la disputa, la elisión y las expresiones del espacio y el tiempo en las perspectivas poscoloniales, los estudios subalternos y otros entendimientos críticos, y analizarlos. 
prudentemente la importancia de la diferencia crítica en un campo tan definido pero enredado.

Finalmente, al asumir las tareas antes esbozadas, los esfuerzos más proféticos han apuntado al lugar y la presencia fundamentales no sólo de protagonistas de élite y heroicos, sino también de sujetos marginales y subalternos - moldeados simultáneamente por las matrices entrecruzadas de género y raza, casta y clase, edad y oficio, comunidad y sexualidad-en la creación de la colonia y la modernidad, del imperio y la nación, de la religión y la política, y del Estado y el ciudadano. Registrar tales sucesos críticos es representar las proposiciones poscoloniales y los estudios subalternos -en constante conversación con la antropología histórica y la teoría social- como participantes-interlocutores en debates actuales más amplios que repiensan la nación-Estado y Occidente, la colonia y la poscolonia y la historia y la modernidad, incluidos especialmente sus atributos socioespaciales-temporales. ${ }^{51}$

Parte de lo que he estado diciendo sobre leer en busca de las posibilidades, así como de las limitaciones de los entendimientos críticos -incluidas las perspectivas decoloniales, poscoloniales y subalternas-, puede aclararse si se considera la obra de Dipesh Chakrabarty, que ofrece destacadas reflexiones sobre la historia y la modernidad, a la vez que expresa cuestiones de espacio y tiempo, implícita y explícitamente. Para empezar, sus escritos han planteado con gran imaginación preguntas clave sobre la presencia de Europa en la historiografía. Interpretando cuidadosamente sus argumentos contra el telón de fondo de la interrogación de Heidegger del artificio de una razón legisladora de sentido, Chakrabarty se ha centrado en la "historia" como un discurso que se produce en los sitios institucionales del mundo académico, y ha dado poderosos argumentos en favor de las formas en que Europa sigue siendo el sujeto teórico soberano de todas las historias. Admitiendo que "Europa" e

${ }^{51}$ Nada de esto pretende negar las tensiones tangibles que abundan en la antropología histórica, en las perspectivas poscoloniales y en los estudios subalternos. En este sentido, por ejemplo, los enfoques que otorgan una primacía analítica a los procesos de economía política y formación del estado se enfrentan a orientaciones que atribuyen un privilegio teórico a órdenes discursivas y regímenes representacionales. Véase Dube, Stitches on Time, op. cit.; Dube, Subjects of Modernity, op. cit. 
"India" son términos "hiperreales" que se refieren a ciertas figuras de la imaginación, Chakrabarty apunta críticamente hacia cómo -en el "mundo fenomenal" de las relaciones diarias de poder-Europa se objetiva y se celebra como el sitio y la escena del nacimiento de lo moderno, actuando como un referente silente que domina el discurso de la historia. Desentrañando las consecuencias de este privilegio rutinario de Europa como pieza central universal de la modernidad y la historia, Chakrabarty revela cómo el pasado y el presente de India o de México -en realidad de todo aquello que no es del todo un Occidente imaginario pero tangible- llegan a representarse en términos de principios irrevocables de fracaso, carencia y ausencia, dado que siempre/ya se les mide contra sucesos evidentes en los ámbitos europeos/euroamericanos. ${ }^{52}$

Estos son resultados de regímenes desarrollistas de tiempo, temporalidad e historia que Chakrabarty enmarca como "historicismo": un penetrante modo de pensar y una manera de conocer que parece íntimamente implicada en entendimientos sociocientíficos y la práctica histórica más amplia. Con base en el principio de "tiempo secular, vacío y homogéneo", el historicismo ha encontrado agudas expresiones desde el siglo XIX, cuando hizo posible "la dominación europea del mundo". ${ }^{53} \mathrm{En}$ este sentido hay cuestionamientos cruciales relacionados con un tiempo singular pero jerarquizante que divide los mundos sociales en espacios "desarrollados" y "retrasados". En verdad, Chakrabarty abre un poco más las preguntas de diferencia histórica, y revela destellos de terrenos temporoespaciales heterogéneos en varias formas: exploraciones del diferimiento-diferencia de una modernidad bengalí en la India colonial; discusiones sobre el tiempo de los dioses y la historiografía; y admisiones de la pluralidad de los mundos vitales contra un historicismo presuntuoso. ${ }^{54}$

${ }^{52}$ Dipesh Chakrabarty, "Postcoloniality and the Artifice of History: Who speaks for 'Indian' Pasts?”, Representations, núm. 37, 1992, pp. 1-26.

${ }^{53}$ Chakrabarty, Provincializing Europe, op. cit.

${ }^{54}$ Dipesh Chakrabarty, "The Difference-deferral of a Colonial Modernity: Public Debates on Domesticity in British Bengal”, en David Arnold y David Hardiman (eds.), Subaltern Studies VIII: Essays in honor of Ranajit Guha, Nueva Delhi, Oxford University Press, 1994, pp. 50-88; Chakrabarty, Habitations of Modernity, op. cit. 
Al mismo tiempo, vale la pena considerar los cierres que acompañan a la apertura de estas preguntas por parte de Chakrabarty. Así, con gran imaginación, intenta "escribir la diferencia en la historia de nuestra modernidad [bengalí/india] en un modo que resista la asimilación de esta historia al imaginario político de las instituciones derivadas de Europa... que dominan nuestras vidas". Sin embargo, Chakrabarty termina por replicar atributos a priori de las principales categorías que se encuentran en el corazón de la "violencia epistémica" que él busca desafiar e interrogar. ${ }^{55}$ Esto se debe a que los ámbitos de género de lo público y lo doméstico, los conceptos clave de personeidad y de lo civil-político, y en realidad de las categorías opuestas de Estado y comunidad, aparentemente derivadas de un esquema maestro de historia moderna, parecen estar siempre ahí, ya existentes, bajo toda modernidad. Aquí hay una interpretación de la diferencia contra, en y por delante de la disciplina. No resulta sorprendente que exactamente este modo de lectura continúe en el esfuerzo de Chakrabarty por recuperar la diferencia de los pasados subalternos (y el tiempo de los dioses y los espíritus) frente a la disciplina de las historias de las minorías (y la obra del historiador radical), y en su intento por expresar la alteridad de "historias necesariamente fragmentarias de pertenencia humana que nunca constituyen un uno o un todo" como existentes junto a la autoridad del historicismo, pero excediéndola. ${ }^{56}$

¿De qué manera se conectan estos elementos con cuestiones del tiempo y el espacio? Considérense ahora el(los) constructivismo(s) generalizado(s), siempre en el aire, que proyecta totalidades y universales como principalmente insustanciales porque se construyen socialmente. Contra estas presunciones, con justa razón Chakrabarty ve universales totalizantes, sus disciplinas y lógicas como elementos que realmente existen. ${ }^{57}$ Sin embargo, cabe preguntar si tal aceptación soslaya la creación de estos universales en relación con particulares, de

\footnotetext{
${ }_{55}$ Chakrabarty, “The Difference-deferral...”, op. cit., p. 84.

${ }^{56}$ Chakrabarty, Habitations of Modernity, op. cit.; Chakrabarty, Provincializing Europe, op. cit., p. 255.

${ }^{57}$ Véase Dipesh Chakrabarty y Saurabh Dube, "Presence of Europe: An Interview with Dipesh Chakrabarty”, en Dube (ed.), Postcolonial Passages, op. cit., pp. 254-262.
} 
totalidades en relación con márgenes, implicando procesos de significado y poder que agudamente producen espacio y tiempo. ¿Estos elementos que leen la diferencia contra, en y por delante de la disciplina - asociando las fabricaciones y producciones mutuas de disciplina y diferencia- resultan en espacios analíticamente segregados? ¿Los atributos sociopolíticos de tales espacios segregados se derivan, posiblemente, de sus bases epistémicas? ¿Chakrabarty cuestiona los términos que exaltan el tiempo homogéneo, pero acepta las rupturas putativas de la modernidad en las que éstos se basan?

\section{Engranajes de los últimos días}

Como me acerco al final de esta narrativa personal, es momento de unir mis aprehensiones inciertas, pero insistentes, del tiempo y el espacio, desentrañadas anteriormente, con los temas de sus entendimientos usuales, representaciones hegemónicas y producciones cotidianas que se plantearon al inicio de este ensayo. En estas consideraciones resulta especialmente importante la producción de espacio-tiempo en la práctica académica como una especie de la actividad diaria. ${ }^{58}$ Tal interpretación, a través de la acción epistemológica, ocurre en un diálogo con aprehensiones rutinarias y hegemónicas del espacio y el tiempo que expresa términos subyacentes de poder y diferencia.

Recuérdese que las primeras formaciones de los estudios subalternos se basaron en representaciones temporales y espaciales dominantes, singulares pero jerarquizantes, que ubicaron a los grupos subalternos (pasivos) y sus culturas gobernantes de dominio (feudales) en tiempos y espacios que estaban detrás de aquellos de la política moderna. Sin embargo, interrogando agudamente la división entre prepolítica y política, se ofrecían igualmente instanciaciones de noveles matrices temporoespaciales, pero sólo una vez los subalternos rompieron los códigos que gobernaban su pasividad, pues ahora había expresiones en-

${ }^{58}$ Sobra decir que tal producción del espacio-tiempo como parte de la práctica epistémica puede seguirse igualmente en relación con tendencias en la antropología histórica que se notaron anteriormente, pero dejaré estos temas para otro tiempo y otro espacio. 
teramente autónomas que no solamente eran contemporáneas de la política democrática moderna, sino que estaban a la vanguardia.

Tal producción del tiempo-espacio como parte de la actividad de creación de conocimiento continuó a través de la oposición más amplia entre comunidad y Estado en los estudios subalternos. Éste fue el caso, ya sea que se demostrara que, a través de medidas principalmente antimodernistas, las valencias temporoespaciales de la modernidad se invirtieron para encontrar comunidades (y fragmentos) arraigadas en la costumbre como triunfantes sobre la nación-Estado (y la historia), ${ }^{59}$ o bien que, recurriendo a la distinción espaciotemporal de Foucault entre la autoridad previa y el poder moderno, las formas de jerarquía de los subalternos indios, basadas en culturas precapitalistas, investigaron las suposiciones hegemónicas de la disciplina historiográfica, ${ }^{60}$ o que la comunidad se colocó en el corazón de la modernidad en formas tales que las virtudes innatas de alteridad y diferencia se convirtieron en antídotos para interminables exaltaciones del capital y el Estado. ${ }^{61}$ En cada caso, los planos espaciotemporales hegemónicos de la modernidad, como plantilla analítica y marcador cronológico, se alcanzaron, pero también se excedieron: ahora se otorgaba a la comunidad, al subalterno, al fragmento y a la diferencia una prioridad ética y política, una precedencia epistemológi-

${ }^{59}$ Pandey, The Construction of Communalism, op. cit.; Gyanendra Pandey, "In Defense of the Fragment: Writing about Hindu-Muslim Riots in India Today", Representations, núm. 37, 1992, pp. 27-55. Estas disposiciones muestran ciertas afinidades con los escritos de Ashis Nandy que han expresado y respaldado sensibilidades anti y contramodernas (que desde luego han sido una parte crítica y formativa de la modernidad durante ya mucho tiempo). La obra de Nandy ha visualizado y expresado la modernidad $-\mathrm{y}$ sus instituciones e imaginaciones asociadas - como señales de un proyecto presuntuoso, constituyente de una colonización de la mente, al mismo tiempo del colonizado y del colonizador. Contra tal colonización hay que colocar los trabajos de la diferencia creativa, la decolonización psíquica y las recuperaciones resueltas de la tradición crítica, en el pasado y en el presente. Ashis Nandy, The Intimate Enemy: Loss and Recovery of Self under Colonialism, Delhi, Oxford University Press, 1983; Ashis Nandy, The Savage Freud and Other Essays on Possible and Retrievable Selves, Princeton, Princeton University Press, 1995; Ashis Nandy, An Ambiguous Journey to the City: The Village and Other Odd Remains of the Self in the Indian Imagination, Delhi, Oxford University Press, 2001.

${ }^{60}$ Chakrabarty, Rethinking Working-Class History, op. cit.

${ }^{61}$ Chatterjee, The Nation and its Fragments, op. cit. 
ca e interpretativa sobre el capital, el Estado-nación, la historia y el poder. Estaba en juego la fabricación epistémica del espaciotiempo, que insinuaba una alteridad por delante de la autoridad como parte de la práctica diaria de los estudios subalternos.

De hecho, estas presunciones y estos protocolos de los estudios subalternos se revelaron dudosamente como vinculadas a disposiciones más amplias de diferencia y poder en entendimientos anti y posfundacionales. Sencillamente, aquí hay orientaciones que muestran al poder — del Estado, la nación, el imperio, la modernidad, el patriarcado o la disciplina- como una totalidad distópica, frecuentemente un enemigo distante. Contra esto, se ofrece el trabajo de la diferencia - de la comunidad, el subalterno, la alteridad, la frontera y el margencomo "particulares no recuperados", siempre un antídoto a las depredaciones de la distopía. ${ }^{62}$ Mucho más que ante una analítica formal, estamos ante estructuras de sensibilidad, tejidos de sentimiento que sujetan las ortodoxias críticas, también subyacente a su producción definida del tiempo y el espacio en la clave cotidiana. ${ }^{63} \mathrm{Si}$ las antinomias de comunidad y Estado en la labor de los estudios subalternos brindan una ilustración, las perspectivas decoloniales ofrecen otro ejemplo apropiado de tales elaboraciones.

Como se analizó, en estas disposiciones el espacio está configurado en formas principalmente delimitadas o relativamente

${ }^{62}$ Primero inicié y abundé en temas que surgen de tales disposiciones en Dube, Stitches on Time, op. cit. Han sido un componente crítico de mi trabajo de investigación, escritura y enseñanza desde entonces.

${ }^{63}$ Una obra en particular que en realidad reconoce la productividad del poder es un ejemplo relevante. Gyan Prakash ha abordado la modernidad como un aparato dominante que engendra alteridad crítica. En su lectura, los términos de la modernidad según se expresan en el trabajo científico encuentran forma y asumen sustancia en la productividad del poder del colonialismo y el nacionalismo. Al mismo tiempo, satisfecho con haber establecido la presencia de la alteridad, Prakash apenas dedica más tiempo a la carga de tal diferencia, particularmente en la poscolonia. Aquí, la autoridad engendra alteridad, pero tal alteridad insinúa un espacio-tiempo intersticial cuya lógica principal es, bueno, ser diferente de la matriz de poder en la que está arraigada. Gyan Prakash, Another Reason: Science and the Imagination of Modern India, Princeton, Princeton University Press, 1999. Por otro lado, la productividad del poder, incluida la "gubernamentalidad", encuentra configuraciones bien definidas en los escritos recientes de Partha Chatterjee, que de nuevo brindan testimonio de la heterogeneidad constitutiva y los énfasis cambiantes de los estudios subalternos. Véase, por ejemplo, Partha Chatterjee, The Politics of the Governed: Reflections on Popular Politics in Most of the World, Nueva York, Columbia University Press, 2004. 
abiertas, y el tiempo puede presentarse como un marco básicamente neutral o un dispositivo colonizador altamente normativo. Aun así, la fuerza ética, epistemológica, política y afectiva de los argumentos en favor de la decolonialidad se derivan de la forma en que producen activamente, como imagen y como práctica, las locaciones morales discretas del "lado subalterno [u otro] de la diferencia colonial" como invariablemente por delante de -con una precedencia de principios y una prioridad a priori sobre- las coordenadas espaciotemporales distópicas de la "modernidad/colonialidad" que buscan abrumar a todos a su paso.

Verdaderamente, como sentimiento, sensibilidad y espíritu, la escisión entre autoridad y alteridad posee una fuerza formidable. Esto también significa que un académico como Dipesh Chakrabarty podría no inclinarse hoy por la disciplina como enemigo distante y ver más allá de la diferencia como esencialmente heroica. No obstante, en la práctica, puesto que se formó una vez en los imaginarios que dividen la disciplina y la diferencia, Chakrabarty debe segregarlos. Aquí se encuentran producciones epistémicas de espacio-tiempo, de alteridad y autoridad que, en realidad, se combinan con frecuencia, pero cuya separación analítica exacta permite que la presencia de la diferencia se lea en/contra las afirmaciones de la disciplina. Así, una heterogeneidad formidable y radical se enfrenta a una singularidad crítica inescapable.

\section{Al final}

Estas consideraciones ponen en primer plano dos conjuntos de preguntas críticas. Primero, ¿qué está en juego al explorar críticamente los términos de poder y del(los) conocimiento(s) dominante(s) sin convertirlos en un terreno totalizado? Los intentos de pluralizar el poder - por ejemplo, la fuerza del colonialismo y el capitalismo, las estipulaciones de la globalización y la modernidad- ¿son meros ejercicios de refinamiento empírico y conceptual de estas categorías? Alternativamente, ¿también implican un "giro ontológico" que no sólo apunta al problema de "qué entidades presuponen" las teorías y las cosmovisiones, 
sino que también cuestiona cuidadosamente a "aquellas 'entidades' presupuestas por nuestras formas típicas de ver y hacer en el mundo moderno?”. ${ }^{64}$ ¿Cuál es el lugar de lo particular, de los "detalles", al desentrañar las determinaciones de poder y diferencia? ${ }^{65}$ ¿Cómo debemos aprender de (pero trascender) ortodoxias críticas más nuevas que interpretan las categorías dominantes como totalidades distópicas? ${ }^{66}$ En pocas palabras, ¿cuáles son los términos y las texturas del entendimiento del poder moldeado por la diferencia o de la autoridad influida por la alteridad?

En segundo lugar, ¿qué distinciones de significado y poder se ponen en primer plano a través de la elaboración de la tradición y la comunidad, de lo local y lo subalterno como categorías oposicionales? ¿Estas categorías en conflicto deben habitar el locus de los "particulares no recuperados", como antídotos a priori a la autoridad, en los espejos de los entendimientos críticos? ${ }^{67}$ ¿Cómo debemos expresar la densa sensualidad y las agudas confusiones de la vida social? ¿De qué maneras puede hacerse esto para no sólo analizar las categorías estereotipadas y los esquemas modulares para ordenar el mundo, sino también para repensar las proyecciones axiomáticas de diferencia resistente que abundan en el aquí y ahora, caracterizando aprehensiones académicas, concepciones comunes e imaginarios neovitalistas? Es decir, ¿qué está en juego al entender la determinación de la diferencia sellada por la productividad del poder, de las formaciones subalternas que portan la marca de los diseños dominantes?

Éstas son algunas preguntas que surgen de cuidadosas consideraciones del tiempo-espacio y sus engranajes con co-

${ }^{64}$ Stephen K. White, Sustaining Affirmation: The Strengths of Weak Ontology in Political Theory, Princeton, Princeton University Press, 2000, pp. 3-4. Considérese también el movimiento hacia una "práctica estratégica de la crítica" en David Scott, Refashioning Futures: Criticism after Postcoloniality, Princeton, Princeton University Press, 1999, pp. 3-10, 17-18.

${ }^{65}$ Certeau, Practice of Everyday Life, op. cit., p. ix.

${ }_{66}$ Tomo prestada esta noción -y la de "particulares no recuperados", que se deriva de la misma- de John McGowan, Postmodernism and its Critics, Ithaca, Cornell University Press, 1991.

${ }^{67}$ Idem. 
nocimiento(s) crítico(s) y formaciones disciplinarias surgidas en la modernidad.

Traducción:

María Capetillo Lozano

Dirección institucional del autor:

Centro de Estudios de Asia y África

El Colegio de México

Carretera Picacho Ajusco 20

Ampliación Fuentes del Pedregal

14110, Ciudad de México

\section{Bibliografía}

Aвrams, Philip, Historical Sociology, Shepton Mallet, Open Books, 1982.

AmIn, Shahid, Event, Metaphor, Memory: Chauri Chaura 1922-1992, Berkeley, University of California Press, 1996.

BANERJEe-Dube, Ishita, "Taming Traditions: Legalities and Histories in Eastern India", en Gautam Bhadra, Gyan Prakash y Susie Tharu (eds.), Subaltern Studies X: Writings on South Asian History and Society, Nueva Delhi, Oxford University Press, 1999, pp. 98-125.

Baxi, Upendra, “'The State's Emissary': The Place of Law in Subaltern Studies", en Partha Chatterjee y Gyanendra Pandey (eds.), Subaltern Studies VII: Writings on South Asian History and Society, Delhi, Oxford University Press, 1992, pp. 257-264.

Bayly, C. A., Indian Society and the Making of the British Empire, Cambridge, Cambridge University Press, 1987.

Bourdieu, Pierre, Outline of a Theory of Practice, Cambridge, Cambridge University Press, 1977.

CerTEAU, Michel de, The Practice of Everyday Life, tr. Steven Rendall, Berkeley, University of California Press, 1984.

Chakrabarty, Dipesh, Habitations of Modernity: Essays in the Wake of Subaltern Studies, Chicago, University of Chicago Press, 2002.

Chakrabarty, Dipesh, "Postcoloniality and the Artifice of History: Who speaks for 'Indian' Pasts?”, Representations, núm. 37, 1992, pp. 1-26. 
Chakrabarty, Dipesh, Provincializing Europe: Postcolonial Thought and Historical Difference, Princeton, Princeton University Press, 2000.

Chakrabarty, Dipesh, Rethinking Working-Class History: Bengal 1890-1940, Princeton, Princeton University Press, 1989.

Chakrabarty, Dipesh, "The Difference-deferral of a Colonial Modernity: Public Debates on Domesticity in British Bengal”, en David Arnold y David Hardiman (eds.), Subaltern Studies VIII: Essays in honor of Ranajit Guha, Nueva Delhi, Oxford University Press, 1994, pp. 50-88.

Chakrabarty, Dipesh y Saurabh Dube, "Presence of Europe: An Interview with Dipesh Chakrabarty", en Saurabh Dube (ed.), Postcolonial Passages: Contemporary History-Writing on India, Nueva Delhi, Oxford University Press, 2004, pp. 254-262.

Chakravorty SPIVAK, Gayatri, "Subaltern Studies: Deconstructing Historiography”, en Ranajit Guha (ed.), Subaltern Studies IV: Writings on South Asian History and Society, Delhi, Oxford University Press, 1985, pp. 330-363.

Chatterjee, Partha, "Introduction: History and the Present", en Partha Chatterjee y Anjan Ghosh (eds.), History and the Present, Delhi, Permanent Black, 2002, pp. 1-23.

Chatterjee, Partha, The Nation and its Fragments: Colonial and Postcolonial Histories, Princeton, Princeton University Press, 1993.

Chatterjee, Partha, The Politics of the Governed: Reflections on Popular Politics in Most of the World, Nueva York, Columbia University Press, 2004.

CoHn, Bernard, "The Command of Language and the Language of Command", en Ranajit Guha (ed.), Subaltern Studies IV: Writings on South Asian History and Society, Delhi, Oxford University Press, 1985, pp. 276-329.

DAs, Veena, "Subaltern as Perspective”, en Ranajit Guha (ed.), Subaltern Studies VI: Writings on South Asian History and Society, Delhi, Oxford University Press, 1989, pp. 310-324.

Dhanagre, D. N., Peasant Movements in India, 1920-1950, Delhi, Oxford University Press, 1983.

Dube, Saurabh, After Conversion: Cultural Histories of Modern India, Nueva Delhi, Yoda Press, 2010.

DubE, Saurabh, "Critical Crossovers: Cultural Identities, Postcolonial Perspectives, and Subaltern Studies", en Margaret Wetherell y Chandra Talpade Mohanty (eds.), The Sage Handbook of Identities, Londres, Sage, 2010, pp. 125-143. 
Dube, Saurabh, Formations of an Evangelical Modernity: Christianity, Conversion, Colonialism 1860-2005, manuscrito, s.f.

Dube, Saurabh, "Introduction: Anthropology, History, Historical Anthropology", en Saurabh Dube (ed.), Historical Anthropology, Nueva Delhi, Oxford University Press, 2007, pp. 1-73.

Dube, Saurabh, "Myths, Symbols, Community”, en Partha Chatterjee y Gyanendra Pandey (eds.), Subaltern Studies VII. Writings on South Asian History and Society, Delhi, Oxford University Press, 1993, pp. 121-156.

Dube, Saurabh, Modernidad e historia, México, El Colegio de México, 2011.

Dube, Saurabh, Native Witness: Colonial Writings of a Vernacular Christianity, manuscrito, s.f.

DuBE, Saurabh, "Peasant Insurgency and Peasant Consciousness", Economic and Political Weekly, vol. 20, núm. 11, 1985, pp. 445-448.

Dube, Saurabh, "Religion, Identity and Authority among the Satnamis in Colonial Central India", tesis doctoral inédita, Cambridge, University of Cambridge.

Dube, Saurabh, "Social History of Satnamis of Chhattigarh", tesis de maestría inédita, Delhi, University of Delhi, 1988.

Dube, Saurabh, Stitches on Time: Colonial Cultures and Postcolonial Tangles, Durham, Duke University Press, 2004.

Dube, Saurabh, Subjects of Modernity: Time-Space, Disciplines, Margins, Manchester, Manchester University Press, 2017.

DubE, Saurabh, "Telling Tales and Trying Truths: Transgressions, Entitlements and Legalities in Village Disputes, Late Colonial Central India", Studies in History, vol. 13, núm. 2, 1996, pp. 171201.

DubE, Saurabh, "Terms that Bind: Colony, Nation, Modernity", en Saurabh Dube (ed.), Postcolonial Passages: Contemporary HistoryWriting on India, Nueva Delhi, Oxford University Press, 2004, pp. 1-37.

Dube, Saurabh, Untouchable Pasts. Religion, Identity, and Power among a Central Indian Community, 1780-1950, Delhi, Vistaar Publications, 1998.

DuBE, Saurabh e Ishita Banerjee-Dube, Unbecoming Modern: Colonialism, Modernity, Colonial Modernities, Nueva Delhi, Social Science Press, 2006.

Dube, Saurabh, Ishita Banerjee-Dube y Edgardo Lander (eds.), Critical Conjunctions: Foundations of Colony and Formations of Modernity, Durham, Duke University Press, 2002.

Dube, Saurabh, Ishita Banerjee-Dube y Walter Mignolo (eds.), Mo- 
dernidades coloniales: Otros pasados, historias presentes, México, El Colegio de México, 2004.

Dussel, Enrique, Ética de la liberación en la edad de la globalización $y$ de la exclusión, Madrid, Trotta, 1998.

Dussel, Enrique, "Europe, Eurocentrism and Modernity (Introduction to the Frankfurt Lectures)", Boundary 2, vol. 20, núm. 3, 1993, pp. 65-76.

Dussel, Enrique, The Invention of the Americas: Eclipse of "the Other" and the Myth of Modernity, Nueva York, Continuum, 1995.

Dussel, Enrique, "Transmodernity", en Saurabh Dube, Ishita Banerjee-Dube y Edgardo Lander (eds.), Critical Conjunctions: Foundations of Colony and Formations of Modernity, Durham, Duke University Press, 2002, pp. 221-244.

FabIan, Johannes, Time and the Other: How Anthropology Makes its Object, Nueva York, Columbia University Press, 1983.

Freitag, Sandria B., Collective Action and Community: Public Arenas and the Emergence of Communalism in North India, Berkeley, University of California Press, 1989.

Genovese, Eugene, Roll, Jordan, Roll: The World the Slaves Made, Nueva York, Pantheon Books, 1978.

Giddens, Anthony, Central Problems in Social Theory: Action, Structure, and Contradiction in Social Analysis, Berkeley, University of California Press, 1979.

GougH, Kathleen, Rural Society in Southeast India, Cambridge, Cambridge University Press, 1981.

Grosfoguel, Ramón, "Decolonizing Post-colonial Studies and Paradigms of Political Economy: Transmodernity, Decolonial Thinking, and Global Coloniality", Transmodernity: Journal of Peripheral Cultural Production of the Luso-Hispanic World, vol. 1, núm. 1, 2011, pp. 1-37.

GuHA, Ranajit, Elementary Aspects of Peasant Insurgency in Colonial India, Delhi, Oxford University Press, 1983.

GuHA, Ranajit, Dominance without Hegemony: History and Power in Colonial India, Cambridge, Harvard University Press, 1997.

GuHA, Ranajit (ed.), A Subaltern Studies Reader: 1986-1995, Minneapolis, University of Minnesota Press, 1997.

GuHA, Ranajit (ed.), Subaltern Studies II: Writings on South Asian History and Society, Delhi, Oxford University Press, 1983.

Hardiman, David, Peasant Nationalists of Gujarat: Kheda District 1917-1934, Delhi, Oxford University Press, 1981.

JHA, Hetukar, Social Structures of Indian Villages: A Study of Rural Bihar, Nueva Delhi, Sage, 1991. 
Koselleck, Reinhart, The Practice of Conceptual History: Timing History, Spacing Concepts, tr. Todd Samuel Presner, Stanford, Stanford University Press, 2002.

Kumar, Ravinder (ed.), Essays on Gandhian Politics: The Rowlatt Satyagraha of 1919, Oxford, Clarendon Press, 1971.

Lefebvre, Henri, The Production of Space, Oxford, Blackwell, 1991. Levinas, Emmanuel, Time and the Other, tr. Richard A. Cohen, Pittsburgh, Duquesne University Press, 1987.

Levinas, Emmanuel, Totality and Infinity: An Essay on Exteriority, tr. Alphonso Lingis, Pittsburgh, Duquesne University Press, 1987.

Levine, Lawrence, Black Culture and Consciousness: Afro-American Folk Thought from Slavery to Freedom, Oxford, Oxford University Press, 1977.

Maldonado-Torres, Nelson, Against War: Vieres from the Underside of Modernity, Durham, Duke University Press, 2008.

McClinTock, Anne, Imperial Leather: Race, Gender, and Sexuality in the Colonial Contest, Nueva York, Routledge, 1995.

McGowan, John, Postmodernism and its Critics, Ithaca, Cornell University Press, 1991.

Mignolo, Walter, "Coloniality at Large", en Saurabh Dube (ed.), Enchantments of Modernity: Empire, Nation, Globalization, Londres, Routledge, 2009, pp. 67-95.

Mignolo, Walter, Local Histories/Global Designs: Essays on the Coloniality of Power, Subaltern Knowledges and Border Thinking, Princeton, Princeton University Press, 2000.

Mignolo, Walter, The Darker Side of Renaissance: Literacy, Territoriality, and Colonization, Ann Arbor, University of Michigan Press, 1995.

Mignolo, Walter, The Darker Side of Western Modernity: Global Futures, Decolonial Options, Durham, Duke University Press, 2011.

Mignolo, Walter, "The Enduring Enchantment (Or the Epistemic Privilege of Modernity and Where to Go from Here)", South Atlantic Quarterly, vol. 101, núm. 4 (número especial editado por Saurabh Dube), 2002, pp. 927-954.

MunN, Nancy, "The Cultural Anthropology of Time: A Critical Essay", Annual Review of Anthropology, vol. 21, 1992, pp. 93-123. Munn, Nancy, The Fame of Gawa: A Symbolic Study of Value Transformation in a Massim (Papua Nerw Guinea) Society, Durham, Duke University Press, 1992.

Nandy, Ashis, An Ambiguous Journey to the City: The Village and 
Other Odd Remains of the Self in the Indian Imagination, Delhi, Oxford University Press, 2001.

Nandy, Ashis, The Intimate Enemy: Loss and Recovery of Self under Colonialism, Delhi, Oxford University Press, 1983.

Nandy, Ashis, The Savage Frend and Other Essays on Possible and Retrievable Selves, Princeton, Princeton University Press, 1995.

O'Hanlon, Rosalind, "Recovering the Subject: Subaltern Studies and Histories of Resistance in Colonial South Asia", Modern Asian Studies, vol. 22, núm. 1, 1988, pp. 189-224.

OrTner, Sherry, "Resistance and the Problem of Ethnographic Refusal", Comparative Studies in Society and History, vol. 37, núm. 1, 1995, pp. 173-193.

PANDEY, Gyanendra, "In Defense of the Fragment: Writing about Hindu-Muslim Riots in India Today", Representations, núm. 37, 1992, pp. 27-55.

Pandey, Gyanendra, The Ascendancy of the Congress in Uttar Pradesh, 1926-1934: A Study in Imperfect Mobilization, Oxford, Clarendon Press, 1978.

Pandey, Gyanendra, The Construction of Communalism in Colonial North India, Delhi, Oxford University Press, 1990.

PraKash, Gyan, Another Reason: Science and the Imagination of Modern India, Princeton, Princeton University Press, 1999.

Quijano, Aníbal, “Colonialidad y modernidad/racionalidad”, Perú Indígena, vol. 13, núm. 29, 1992, pp. 11-21.

Quijano, Aníbal, "Coloniality of Power, Ethnocentrism, and Latin America”, Nepantla, vol. 1, núm. 3, 2000, pp. 533-580.

Quijano, Aníbal, "La colonialidad del poder y la experiencia cultural latinoamericana”, en Roberto Briceño-León y Heinz R. Sonntag (eds.), Pueblo, época y desarrollo: la sociología de América Latina, Caracas, Nueva Sociedad, 1998, pp. 139-155.

RabinOvich, Silvana, "Alterity”, en Robert McKee Irwin y Mónica Szurmuk (eds.), Dictionary of Latin American Cultural Studies, Gainesville, University Press of Florida, 2012, pp. 17-22.

Redfield, Robert, Peasant, Society, and Culture, Chicago, University of Chicago Press, 1956.

SARKAR, Sumit, Writing Social History, Delhi, Oxford University Press, 1997.

Scotт, David, Refashioning Futures: Criticism after Postcoloniality, Princeton, Princeton University Press, 1999.

Shohat, Ella, "Notes on the Post-colonial", en Padmini Mongia (ed.), Contemporary Postcolonial Theory: A Reader, Londres, A Hodder Arnold Publication, 1996, pp. 321-334. 
SIDDIQI, Majid, Agrarian Unrest in North India: The United Provinces, 1918-1922, Nueva Delhi, Vikas, 1978.

Skaria, Ajay, "Writing, Orality, and Power in The Dangs, Western India, 1800s-1920s", en Shahid Amin y Dipesh Chakrabarty (eds.), Subaltern Studies IX: Writings on South Asian History and Society, Delhi, Oxford University Press, 1996, pp. 13-58.

Skaria, Ajay, Hybrid Histories: Forests, Frontiers and Wildness in Western India, Nueva Delhi, Oxford University Press, 1999.

Soja, Edward, Postmodern Geographies: The Reassertion of Space in Critical Social Theory, Londres, Verso, 1989.

Thomas, Nicholas, Out of Time: History and Evolution in Anthropological Discourse, Cambridge, Cambridge University Press, 1989. Thompson, E. P., Customs in Common, Nueva York, New Press, 1991.

WHITE, Stephen K., Sustaining Affirmation: The Strengths of Weak Ontology in Political Theory, Princeton, Princeton University Press, 2000. 